\section{ANÁLISIS BIBLIOMÉTRICO DE LAS REVISTAS ESPAÑOLAS DE PREHISTORIA Y ARQUEOLOGÍA EN LOS ÚLTIMOS DIEZ AÑ̃S}

\author{
BIBLIOMETRIC ANALYSIS OF SPANISH \\ JOURNALS ON PREHISTORY AND \\ ARCHAEOLOGY DURING THE LAST \\ DECADE
}

\author{
ÁNGEL L. RODRÍGUEZ ALCALDE (*) \\ JOSE MARÍA SÁNCHEZ NISTAL (**) \\ MARÍA ISABEL MARTÍNEZ \\ NAVARRETE $(* * *)$ \\ MARÍA JESÚS SAN MILLÁN \\ BUJANDA (**)
}

\section{RESUMEN}

El artículo da cuenta de ciertos aspectos de la actividad científica en Prehistoria y Arqueología a través del análisis de citas desde revistas españolas entre otros factores. Plantea las dificultades derivadas del incumplimiento sistemático de la periodicidad y la notable falta de normalización en la recogida de referencias bibliográficas por parte de las revistas, lo que dificulta la participación de las publicaciones españolas en los circuitos internacionales de citas y contribuye al ostracismo de la producción científica española. Se ofrecen datos sobre coautoría, temática y lengua. Se analiza la importancia del territorio advirtiendo que entre las re-

(*) Departamento de Prehistoria. Facultad de Geografía e Historia. Universidad Complutense de Madrid. Ciudad Universitaria, s/n. 28040 Madrid.

(**) Unidad de Documentación en Ciencias Humanas. CINDOC. CSIC. Pinar, 25. 28017 Madrid.

(***) Departamento de Prehistoria. Centro de Estudios Históricos. CSIC. Serrano, 13. 28001 Madrid. E-mail: cehse1 d@fresno.csic.es.

El artículo fue remitido en su versión final el 20-III-96. vistas citadas la intensidad de citas decrece con la distancia a la región de publicación. Asimismo, se valora el interés de los análisis de citas como criterio metodológico en la evaluación de las publicaciones periódicas. Se concluye que el localismo provocado por la orientación descriptiva de la investigación tiene dos graves riesgos: 1) la compartimentación de la comunidad científica en grupos desconectados y 2) una conceptualización actualista del pasado.

\begin{abstract}
The paper explains some issues concerning scientific activity in Prehistory and Archaeology through the citation analysis from Spanish periodicals and other factors. It states the difficulties caused by the journals' systematic non-observance of their periodicity and their noticeable lack of bibliographical citation stardardization. That situation harms the contribution of Spanish journals to the international networks of citation and leads to ostracism
\end{abstract}


of Spanish scientific production. Some data concerning co-authorship, theme and language are offered. The analysis of the importance of territory in the cited journals shows that the number of citations diminishes when the distance to the place of publication increases. The relevance of citation analysis as a methodological criteria for periodicals evaluation is also considered. It is concluded that the localism provoked by the descriptive trend of archaeological research has two serious risks: 1) the division of the scientific community into disconnected groups, and 2) the use of very contemporary demarcation lines as a relevant tool for the study of the past.

Palabras clave: Arqueología. Prehistoria. Artículos de revistas. Bibliometría. Análisis de citas. Publicaciones periódicas. Metodología. Factor de Impacto. España.

Key words: Archaeology. Prehistory. Journal articles. Bibliometry. Citation analysis. Periodicals. Methodology. Impact Factor. Spain.

\section{INTRODUCCIÓN}

En la actualidad prácticamente todas las áreas científicas utilizan con éxito los estudios cuantitativos como método para progresar en el conocimiento. Durante los últimos años, la "sociología de la ciencia" ha supuesto una seria revisión de los mismos y la bibliometría (a veces llamada cienciometría) se ha configurado como "aquella disciplina que analiza estadísticamente una determinada literatura científica, su evolución histórica, sus campos y desarrollos temáticos, así como sus autores y usos en el ámbito científico propio o próximo" (Ferreiro, 1993: 18). Conceptos como cita, vida media, indicadores de calidad, factor de impacto, coautoría, redes de colaboración, difusión del conocimiento, etc., se han incorporado ya como indicadores del desarrollo de una materia.

En bibliometría los parámetros más generalmente admitidos por su objetividad se derivan del análisis de las citas bibliográficas en revistas. Sus resultados se consideran indicativos de la calidad de estas últimas y se utilizan para que los científicos puedan planificar su investigación y sus publicaciones de forma más racional al conocer la opinión de sus colegas al respecto. Además, los editores y responsables de subvenciones pueden mejorar y mantener la calidad de sus publicaciones más efectivamente al saber cómo son evaluadas y las bibliotecas pueden obtener datos objetivos para el sostenimiento de las publicaciones nucleares. Muy extendidos en los campos de las ciencias puras y tecnológicas, los análisis bibliométricos están ampliándose ahora a las Humanidades y Ciencias Sociales (Abt, 1992; Finkenstaedt, 1990).

La relevancia de los "rankings" de citas ha sido cuestionada por varios autores, pero al menos se puede coincidir con Singleton (1976: 258-259) en que "las citas aportan una medida cuantitativa de 'algo', y que el debate que debe mantenerse justamente, es el de averiguar qué es ese 'algo"'. En ese sentido, extraer consecuencias de la comparación de los datos obtenidos en $\mathrm{Hu}$ manidades y en ciencias puras puede deslizar errores conceptuales en los análisis de carácter finalista, errores ajenos al tratamiento científico de la documentación. Para vigilarlos, son importantes y deben fomentarse los estudios de citas aplicados a áreas concretas del saber mediante la descripción de modelos, perfiles o pautas de publicación de su literatura, en un momento dado o a través del tiempo. En esta tarea es recomendable la conjunción de equipos de documentalistas y especialistas en la materia que se trate.

Este artículo se propone dar cuenta de ciertos aspectos de la actividad científica española en Prehistoria y Arqueología a través de una investigación sobre el conocimiento y uso que de las publicaciones tienen los autores a partir de las citas y de los artículos de una muestra de revistas españolas (Rodríguez et alii, e.p.).

La oportunidad de un estudio de esas características viene dada por el escaso desarrollo en aquellas disciplinas de la bibliometría, que se ha demostrado tan relevante en otras áreas de conocimiento. Además, quiere ser una llamada a la reflexión sobre el perjuicio que, para la investigación arqueológica española, supone que el grado de cobertura de sus revistas en las bases y repertorios internacionales sea mínimo cuando no inexistente. Así, en el Ulrich's, uno de los más difundidos y que recoge más de 68.000 títulos de revistas, el porcentaje de las revistas españolas de Historia sobre el total mundial es de 1,63 (Sánchez Nistal, 1995: 561-562). Los estudios sobre el

T. P., 53, n. ${ }^{\circ} 1,1996$ 
impacto de la investigación española en Historia Antigua apuntan en este mismo sentido (Remesal et alii, 1991).

La validez del análisis bibliométrico depende de la significación, tanto del tipo de documento seleccionado, en este caso los artículos, como de la definición de la muestra. En cuanto a la consideración del artículo como representativo de la investigación en un área, baste decir que la publicación periódica es actualmente el vehículo más utilizado en la difusión del conocimiento científico. Ya en 1975 se publicaban dos millones de artículos firmados al año (Sales et alii, 1995: 1). La muestra que hemos seleccionado viene avalada por la opinión de los expertos.

\section{LA MUESTRA}

\subsection{Definición}

La muestra es la clave de la calidad de los trabajos estadísticos. Para determinarla, enviamos a los comités de redacción de las 73 revistas españolas con las que Trabajos de Prehistoria tiene intercambio una relación donde figuraban todas ellas solicitando que marcaran y/o, en su caso, añadieran las que consideraran más relevantes. Las condiciones fijadas para la selección eran que la representatividad territorial e institucional quedara garantizada y que tuvieran al menos diez años de antigüedad. Obtuvimos 47 respuestas con 102 títulos de revistas.

\begin{tabular}{|c|c|c|c|c|c|c|c|c|c|c|c|c|c|c|c|c|c|c|c|c|c|c|c|c|}
\hline & A & B & C & D & $\mathrm{E}$ & $\mathrm{F}$ & G & H & I & $\mathrm{J}$ & $\mathrm{K}$ & $\mathrm{L}$ & M & N & 0 & $\mathrm{P}$ & Q & $\mathrm{R}$ & s & $\mathrm{T}$ & $\mathrm{U}$ & $\mathrm{V}$ & $\mathrm{x}$ & Y \\
\hline AEspA (88-93) & 6 & 129 & 2.568 & 1.319 & 207 & 942 & 258 & 23 & 8 & 1 & 17 & 240 & 123 & 51 & 52 & 11 & 2 & 1 & & 934 & 51 & 143 & 14,7 & 19,9 \\
\hline $\operatorname{APL}(81-90)$ & 5 & 123 & 2.112 & 1.325 & 192 & 540 & 180 & 19 & 13 & 4 & 31 & 215 & 125 & 33 & 49 & 3 & 5 & & & 485 & 33 & 147 & 19,8 & 17,17 \\
\hline Boletin. Museo Zaragoza (88-92) & 4 & 27 & 271 & 138 & 56 & 91 & 37 & & 4 & & 1 & 35 & 12 & 8 & 13 & & 2 & & & 63 & 15 & 17 & 36,8 & 10,03 \\
\hline$B S A A(89-93)$ & 5 & 70 & 1.587 & 919 & 159 & 499 & 147 & 8 & & 6 & 8 & 128 & 72 & 22 & 22 & 1 & 8 & 1 & 2 & 537 & 51 & 110 & 40,3 & 22,67 \\
\hline Caesaraugusta (87-92) & 5 & 49 & 1.108 & 476 & 124 & 496 & 108 & 2 & 13 & 9 & 4 & 130 & 55 & 21 & 46 & & 4 & & 4 & 160 & 29 & 15 & 25,8 & 22,61 \\
\hline CuPAUAM (88-92) & 5 & 72 & 1.990 & 1.058 & 178 & 706 & 182 & 27 & 13 & & 4 & 165 & 75 & 33 & 38 & 12 & 6 & 1 & & 143 & 27 & 31 & 25 & 27,63 \\
\hline Cuad. Preh. Gr. (83-88) & 5 & 72 & 1.262 & 777 & 115 & 310 & 136 & 19 & 15 & & 5 & 147 & 104 & 15 & 16 & 5 & 7 & & & 432 & 65 & 151 & 28 & 17,52 \\
\hline QUAD PREH.CAST. (81-86) & 5 & 60 & 895 & 609 & 115 & 185 & 74 & 3 & 6 & 1 & 17 & 103 & 73 & 10 & 12 & 2 & 5 & 1 & & 237 & 45 & 98 & 20,6 & 14,91 \\
\hline Cypsela (87-93) & 5 & 87 & 1.268 & 798 & 131 & 263 & 110 & 40 & 36 & 2 & 19 & 235 & 158 & 26 & 23 & 10 & 11 & 7 & & 200 & 49 & 90 & 12,2 & 14,57 \\
\hline Empúries (79-89) & 6 & 182 & 2.406 & 1.327 & 217 & 764 & 251 & 23 & 19 & 4 & 18 & 435 & 275 & 56 & 83 & 9 & 7 & 4 & 1 & 724 & 55 & 145 & 11,9 & 13,21 \\
\hline$E A A(86-93)$ & 5 & 26 & 1.157 & 788 & 116 & 224 & 133 & & & 2 & 10 & 38 & 23 & 11 & 4 & & & & & 394 & 33 & 154 & 91,9 & 44,5 \\
\hline $\operatorname{EAE}(90-94)$ & 14 & 38 & 1.536 & 1.014 & 165 & 351 & 157 & 8 & 4 & 1 & 1 & 143 & 93 & 22 & 23 & 3 & 2 & . & & 882 & 74 & 82 & & 40,42 \\
\hline Munibe (90-94) & 5 & 93 & 1.744 & 1.173 & 145 & 392 & 141 & 23 & 6 & & 9 & 283 & 191 & 32 & 52 & 5 & 1 & 2 & & 523 & 83 & 305 & 13 & 18,75 \\
\hline Pyrenae (79-93) & 5 & 101 & 1.281 & 732 & 174 & 339 & 154 & 21 & 25 & 2 & 8 & 150 & 74 & 37 & 22 & 7 & 9 & 1 & & 360 & 23 & 43 & 15,1 & 12,68 \\
\hline SAGVNTVM $(90-94)$ & 5 & 70 & 1.723 & 1.013 & 182 & 469 & 190 & 30 & 17 & 1 & 3 & 175 & 93 & 35 & 34 & 4 & 7 & 1 & 1 & 432 & 57 & 87 & 15,8 & 24,61 \\
\hline$T P(80-93)$ & 14 & 240 & 5.437 & 3.268 & 305 & 1.505 & 532 & 72 & 34 & 1 & 25 & 431 & 251 & 57 & 85 & 25 & 11 & 2 & & 961 & 102 & 339 & 19,1 & 22,65 \\
\hline Zephyrus (80-92) & 9 & 306 & 5.131 & 3.108 & 297 & 1.425 & 478 & 62 & 39 & 8 & 11 & 536 & 310 & 82 & 97 & 24 & 19 & 1 & 3 & 679 & 68 & 243 & 21,4 & 16,76 \\
\hline Totales & 108 & 1.745 & 33.474 & $\mid 19.842$ & 2.878 & 9.501 & 3.268 & 380 & 252 & 42 & 191 & 3.589 & 2.107 & 551 & 671 & 121 & 106 & 22 & 11 & 8.146 & 860 & 2.200 & & \\
\hline
\end{tabular}

Cuadro 1. Archivo Español de Arqueología (AEspA), Archivo de Prehistoria Levantina (APL), Boletín del Seminario de Estudios de Arte y Arqueología (BSAA), Cuadernos de Prehistoria de la Universidad de Granada (Cuad.Preh.Gr.), Cuadernos de Prehistoria y Arqueología de la Universidad Autónoma de Madrid (CuPAUAM), Cuadernos de Prehistoria y Arqueología Castellonenses (QUAD.PREH.CAST.), Estudios de Arqueología Alavesa (EAA), Excavaciones Arqueológicas en España (EAE), Trabajos de Prehistoria (TP).

A: Números vaciados: B: Artículos vaciados: C: Número total de citas generadas. D: Número de citas a revistas. E: Número de revistas distintas citadas. F: Citas a Monografías. G: Citas a Congresos. H: Citas a Tesis Doctorales. I: Citas a Memorias de Licenciatura. J: Citas a Informes. K: Citas a Otros. L: Autocitas de autor emitidas. M: Autocitas de autor emitidas a revistas. N: Autocitas de autor emitidas a Congresos. O: id. a Monografías. P: $i d$. a Tesis. Q: id. a Memorias de Licenciatura. R: $i d$. a Otros. S: $i d$. a Informes. T: Citas totales recibidas. U: Autocitas de autor recibidas. V: Autocitas a la propia revista. X: Páginas por artículo (P/A). Y: Media de citas españolas por artículo. 
Se comenzó la toma de datos por los diez últimos años de diversas revistas (Cuadro 1). Los resultados del análisis pusieron de manifiesto que los cinco últimos años garantizaban la representatividad de la muestra. La falta de correspondencia entre años y números vaciados fue la principal dificultad que hubo que afrontar. Por otra parte, la serie Excavaciones Arqueológicas en España, muy valorada por los investigadores (Anexo) y cuyos datos aparecen en el cuadro 1, fue excluida de algunos apartados del estudio general por su carácter monográfico. El trabajo se centró en el análisis y recogida de las citas incluidas en los artículos de los cinco últimos números publicados de 16 revistas tomando como tope retrospectivo el año 1979.

Se establecieron dos bloques de documentos citados por su lugar de publicación: España y otros países. El estudio partió de las citas hechas a publicaciones españolas desde 1.745 artículos. La base de datos tiene 33.476 referencias. Hay citas a 837 revistas distintas, de las que 324 únicamente reciben una cita y 68 aparecen debido a autocitas de autor. En las revistas de la muestra la proporción de citas españolas - todas las del Estado- sobre extranjeras es aproximadamente del $61,37 \%$.

En el anexo están las 107 revistas y series monográficas que han recibido al menos 30 citas en la muestra. Sobre esta lista hay que advertir: 1) que es un "ranking" de citas absolutas, es decir, pueden estar penalizadas las revistas más jóvenes; y 2) que incluye publicaciones seriadas por su relevancia en la investigación reciente.

Se recogieron citas a siete tipos de documentos:

«Revistas y Series monográficas»: los congresos publicados en revistas, los anejos y los números con un solo documento se consideran revistas. El empleo del mismo tratamiento tipográfico para éstas y las Series ha imposibilitado en muchos casos su diferenciación. Por otro lado, las segundas también se citan por el título de la monografía, por ejemplo Scripta Praehistorica. Francisco Jordá Oblata es Acta Salmanticensia, 156.

«Monografías»: incluyen todas las citas que sólo tienen título y año, los catálogos, las mis- celáneas y los homenajes, excepto que se hayan organizado como congresos.

«Congresos»: las publicaciones de actas de reuniones científicas salvo cuando aparecen en revistas, si bien no siempre se facilita esa información.

«Tesis» $\mathrm{y}$ «Memorias de Licenciatura»: sólo inéditas. Las publicadas se citan por el tipo de documento correspondiente.

«Informes»: inéditos depositados en la Administración e informes técnicos manejados por los autores.

«Otros»: guías, memorias de actividades, adquisiciones, diccionarios, enciclopedias, discursos, conferencias, programas de fiestas, folletos, y publicaciones escolares inéditas o no.

La casuística anterior ya insinúa los graves problemas en la realización de una base de datos con las varias decenas de miles de documentos exigibles para un análisis bibliométrico concluyente. Algunos obstáculos se deben al hábito y la forma de citar de los investigadores, a la precariedad de medios materiales y humanos de los editores de publicaciones periódicas, en su mayoría instituciones, y a la desidia de los responsables científicos de su edición. Todo esto se manifiesta, respecto a los primeros, en la aparición de citas incompletas y erróneas que sugieren la falta de un conocimiento directo de la fuente. La cita bibliográfica combina diversas formas de llamada en el texto (año y autor; $o p$. cit., ibidem, nota al pie) con diversas localizaciones en el mismo. La heterogeneidad de la referencia introduce errores en la cuantificación.

La falta de normalización en periodicidad, estructura y aspectos formales en revistas y series expresa la poca importancia que, en último término, les prestan sus instituciones editoras. Pareciera que la escasa dotación de medios materiales se correlacionara con una falta de atención a la profesionalidad de las publicaciones que repercute, también, en los comités de redacción. Por otro lado, la ya comentada ausencia de las mismas en bases de datos internacionales se explica también por la práctica exclusividad de editores institucionales, con menor sensibilización hacia la explotación comercial que los privados que sí fomentan el desarrollo de una política de difusión de sus publicaciones hacia cauces más amplios (Sánchez Nistal, 1995: 563).

T. P., 53, n. ${ }^{\circ} 1,1996$ 


\subsection{Aspecto técnico-estadístico}

Para la recogida de datos se realizó una aplicación en dBase IV (1) que supuso el diseño de tres bases de datos relacionadas entre sí, de forma que la utilización del sistema por parte del usuario era única.

Una primera base fuente recogía los datos catalográficos de los artículos vaciados: título de la revista, volumen, número, año, primera y última página, autor y número de colaboradores.

Una segunda base de revistas registraba el título de cualquier publicación periódica citada en los artículos fuente, guardándolo en memoria de manera que si volvía a necesitarse bastaba su identificación en esta base para incorporarlo a la cita. Este sistema permitía cotejar los títulos de las revistas citadas que aparecen de muy distintas formas en los artículos fuente y recogerlos de forma unívoca. La base de revistas citadas se concibió para poder consultar rápidamente las aparecidas e incorporar en el momento las nuevas de forma normalizada.

Por fin, una tercera base de datos, la más compleja, permitía recoger individualmente cada una de las citas españolas de los artículos vaciados. Para ello el sistema preguntaba primero por el tipo de documento citado. En todos los casos, introducía el año citado y si era una autocita de autor. Cuando era una cita a revista española, se registraba su título homologado a través de la segunda base de datos y el programa contabilizaba automáticamente las autocitas de la propia revista. El programa incorporaba una serie de validaciones y pedía confirmación de algunos datos para evitar errores.

Para todos los datos recogidos (Cuadro 1) se utilizó dBase IV en el cálculo de las clasificaciones jerarquizadas, listados de trabajo, conteos, etc., de manera global e individualizada por revistas y años vaciados.

Se utilizaron tres equipos informáticos independientes: al principio, máquinas $i 386$ y posteriormente un $i 486$ y dos $i 386$. Obviamente, cuando la base de revistas citadas fue adqui-

(1) La aplicación fue diseñada por Gregorio de Vicente (CINDOC). La recogida de datos la llevaron a cabo los autores del artículo y Juan Carlos García Santos. riendo un volumen considerable, el equipo utilizado era determinante en cuanto a la rapidez de respuesta del sistema. Los tres equipos trabajaron de forma autónoma reuniéndose periódicamente para unificar la recogida de los datos. Para el proceso final se utilizó un Pentium 90.

\section{TIPOS DE DOCUMENTOS Y DIFUSIÓN DE LA INVESTIGACIÓN}

\subsection{Las citas a publicaciones extranjeras}

Las citas a publicaciones extranjeras son el $38,62 \%$. Su proporción respecto a las españolas (2) así como la conexión entre tipo de documento e idioma se aborda a partir del último volumen de las revistas de la muestra (Cuadro 2). Las 3.684 referencias extranjeras

\begin{tabular}{|l|r|r|r|r|r|r|r|r|r|}
\hline & Francés & Inglés & Alemán & Portugués & Italiano & Español & Otros & Total & $\%$ \\
\hline Ar & 702 & 518 & 144 & 71 & 170 & 67 & 11 & 1.683 & 45,7 \\
\hline Mo & 586 & 599 & 207 & 37 & 207 & 77 & 7 & 1.720 & 46,7 \\
\hline Co & 97 & 30 & 2 & 12 & 22 & 34 & 4 & 201 & 5,5 \\
\hline Te y ML & 11 & 8 & & & & & & 19 & 0,5 \\
\hline In & & 1 & & & & & & 1 & \\
\hline Ot & 24 & 11 & 11 & 6 & 4 & 1 & 3 & 60 & 1,6 \\
\hline Total & 1.420 & 1.167 & 364 & 126 & 403 & 179 & 25 & 3.684 & \\
\hline$\%$ & 38,5 & 31,7 & 9,9 & 3,4 & 10,9 & 4,9 & 0,7 & & \\
\hline
\end{tabular}

Cuadro 2. Idiomas por tipo de documento en el último volumen de las revistas de la muestra.

Ar: Revistas y Series monográficas. Mo: Monografías. Co: Congresos. Te: Tesis. ML: Memorias de licenciatura. In: Informes. Ot: Otros. El epígrafe "Español" corresponde a los documentos en este idioma publicados fuera de España.

de esta base de datos son suficientes para iniciar una aproximación global a la permeabilidad de la investigación arqueológica y precisar el ámbito territorial de las publicaciones (apartado 5). En cuanto al estudio por revistas, sólo el caso de Empúries merece tratarse con cautela ya que representa la producción de cuatro años.

(2) Madrider Mitteilungen y Mélanges de la Casa de Velázquez se han incluido en el estudio de citas españolas. 


\begin{tabular}{|c|c|c|c|c|c|c|c|c|c|c|c|c|c|c|c|c|c|c|c|c|c|c|c|c|c|c|c|c|c|c|c|c|}
\hline Lengua & & & Fran & & & & & & Ingle & & & & & & mán & & & Italia & & & & Portug & & & & Españ & & & & Otros & & \\
\hline Tipo de documento & $\mathrm{Ar}$ & Mo & $\mathrm{Co}$ & $\mathrm{Te}$ & $\mathrm{ML}$ & $0 t$ & $\mathrm{Ar}$ & Mo & $\mathrm{Co}_{0}$ & $\mathrm{Te}$ & In & $\mathrm{Ot}_{\mathrm{t}}$ & Ar & Mo & $\mathrm{Co}_{\mathrm{L}}$ & $\mathrm{ot}$ & $\mathrm{Ar}$ & Mo & $\mathrm{Co}_{0}$ & $\mathrm{Ot}$ & $\mathrm{Ar}$ & Moc & Co & $\mathrm{Ot}$ & $\mathrm{Ar}$ & Mo & \begin{tabular}{c|c|c} 
Co & \\
\end{tabular} & \begin{tabular}{l|l}
$\mathrm{Ot}$ & $\mathrm{A}$ \\
\end{tabular} & \begin{tabular}{l|l}
$\mathrm{Ar}$ & $\mathrm{M}$
\end{tabular} & Mo & \begin{tabular}{c|l}
$C_{0}$ & 0 \\
0
\end{tabular} & $\%$ \\
\hline AEspA (1993) & 35 & 66 & 9 & & & 2 & 34 & 61 & 5 & & & 1 & 9 & 19 & & 4 & 3 & 1 & & & 37 & 56 & 7 & 2 & 8 & 4 & 4 & & & & 1 & 141,8 \\
\hline$A P L(1990)$ & $70 \mid$ & 29 & 6 & & & 5 & 24 & 30 & 1 & & & 1 & 19 & 5 & & & 2 & 2 & & & 3 & 2 & & & & 7 & 2 & & & & & 37,2 \\
\hline Boletin. Musso Zaragoza (1992) & $29 \mid$ & 3 & 3 & & & & 6 & 2 & & & & & & & & & & & & & 3 & 1 & 1 & & 1 & & & & & & & 42,9 \\
\hline$B S A A(1993)$ & 13 & 18 & 4 & & & & 6 & 12 & & & & & 7 & 8 & & & 9 & 8 & & & 4 & 4 & 2 & & 1 & 2 & & & & & & 19,3 \\
\hline Caesaraugusta (1992) & 44 & 32 & 1 & 1 & & & 47 & 43 & 4 & 1 & & & 21 & 18 & & 1 & 1 & 2 & 2 & & 8 & 21 & & & 3 & & & & & 2 & & 64,7 \\
\hline CuPAUAM (1992) & 31 & 29 & 6 & & & 1 & 64 & 51 & 2 & & & & 5 & 13 & & & 12 & 8 & 5 & 6 & 14 & 15 & & & 8 & 4 & 1 & 1 & & & & 46,3 \\
\hline Cuad. Preh. Gr. (1988) & 7 & 15 & 1 & & & & 22 & 22 & 2 & 1 & & & 3 & 11 & & & 1 & & 1 & & 1 & 2 & 1 & & 2 & 2 & & & & & & 30,6 \\
\hline QUAD PREH. CAST. (1986) & $48 \mid$ & 30 & 7 & 2 & & & 22 & 14 & 3 & & & & 12 & 10 & 2 & & 1 & & & & 13 & 5 & 1 & & & & & & 6 & & & 49,3 \\
\hline Cypsela (1993) & 32 & 29 & 6 & & & 1 & 21 & 27 & & & & & & 5 & & & 1 & 2 & & & 9 & 12 & & & 3 & 2 & & & & 1 & & 43 \\
\hline Empúries (1989) & 141 & 131 & 21 & 2 & & 9 & 87 & 115 & 5 & 1 & 1 & 5 & 36 & 48 & & & 14 & 4 & 1 & & 55 & 56 & 6 & 1 & 18 & 28 & 7 & & 2 & 1 & 2 & 140,5 \\
\hline$E A A(1993)$ & & 5 & & & & & & 2 & & & & & & 1 & & & & & & & & 1 & & & & & & & & & & 16,6 \\
\hline Munibe (1994) & 77 & 21 & 2 & 3 & 1 & 1 & 27 & 12 & & 2 & & 1 & 1 & & & & & & 1 & & 1 & & & & & 2 & & & & & & \begin{tabular}{l|l}
1 & 49
\end{tabular} \\
\hline Pyrenae (1993) & 59 & 31 & 8 & & & & 26 & 25 & 4 & & & & 3 & 5 & & & & & & & 8 & 6 & 3 & & 5 & 9 & 7 & & 2 & & & 36,6 \\
\hline SAGVNTVM (1994) & 34 & 47 & 8 & & & 3 & 16 & 25 & 1 & & & 1 & 7 & 12 & & 3 & 5 & & & & 8 & 14 & 1 & & 4 & 4 & & & 1 & 2 & & 30 \\
\hline$T P(1993)$ & $19 \mid$ & 31 & 2 & & & 1 & 84 & 119 & 2 & 3 & & 2 & 8 & 28 & & & 12 & 3 & & & 2 & 2 & & & 4 & 5 & 4 & & & & & 45,7 \\
\hline Zephynus (1992) & 63 & 69 & 13 & & & 1 & 32 & 39 & 1 & & & & 13 & 24 & & 3 & 10 & 7 & 2 & & 4 & 10 & & 1 & 10 & 8 & 8 & & & 1 & 1 & 26,6 \\
\hline
\end{tabular}

Cuadro 3. Totales por idiomas y tipo de documento en el último volumen de las revistas de la muestra (cft. Cuadro 2).

El $70 \%$ de las citas son a obras en francés e inglés cuya temática abarca desde la Prehistoria hasta la Historia Moderna. Las siguientes lenguas más citadas son el alemán y el italiano que podemos vincular con la arqueología clásica y medieval (Salvatierra, 1990: 39-40). Tambien hay que tener en cuenta el influjo de instituciones extranjeras como la Casa de Velázquez (Gran-Aymerich y Gran-Aymerich, 1991) y el Instituto Arqueológico Alemán tanto en relación con esos estudios como con la investigación prehistórica (Grünhagen, 1979; Schubart, 1995). En conjunto italiano, alemán y portugués no superan el $25 \%$. Las referencias a publicaciones en español editadas fuera de España rondan el 5\%, mientras otras lenguas no llegan al $1 \%$ y sólo están presentes en siete revistas.

El $90 \%$ de los documentos son monografías y artículos, siendo las primeras más citadas que los segundos excepto en la lengua francesa. La comparación con los documentos españoles análogos es improcedente debido a las diferencias en la muestra (apartado 3.2).

El estudio por revistas de las citas extranjeras muestra que la mayoría ronda la media $(38,62 \%)$ (Cuadro 3$)$. Por encima y por debajo aparecen revistas con valores muy similares pero de muy diferente ámbito territorial: de carácter general como Trabajos de Prehistoria (45,7\%) y Zephyrus $(26,6 \%)$, y regional como Cypsela (43\%) y Estudios de Arqueología Alavesa $(16,6 \%)$. Los valores extremos están representados por esta última y Caesaraugusta $(64,7 \%)$ y, a juzgar por la media ponderada $(38,49 \%)$, son irrelevantes en la configuración de la muestra.

La interpretación de la citas debe tener en cuenta, primero, los artículos referidos a materiales o yacimientos arqueológicos extranjeros; en segundo lugar, aquellos cuya temática requiere o aconseja una amplia contextualización espacial (Paleolítico, Colonizaciones, Romano/Paleocristiano, Epigrafía/Numismática...) o bibliográfica (Teoría y Metodología); y, en tercer lugar, la proximidad de la zona de estudio a las fronteras actuales.

En cada volumen estos factores aparecen aislados o asociados de distinta forma. Así, por ejemplo, la mitad de los artículos de Cypsela y

T. P., 53, n. ${ }^{\circ} 1,1996$ 
todos los de Caesaraugusta menos uno, son de Arqueología Clásica incluyendo en esta última dos sobre Gerasa (Jordania). Empúries está en una situación similar ya que del total de 79 artículos, 34 son de estudios clásicos y otros 16 tratan cuestiones de otros países tanto por autores extranjeros como nacionales. Por su parte, el contenido descriptivo del volumen de Estudios de Arqueología Alavesa y del Boletín del Seminario de Estudios de Arte y Arqueología reduce significativamente la cita extranjera. Munibe, en cambio, publica tres artículos en lengua francesa sobre el Suroeste francés y uno en inglés de un total de 13. Si a ello añadimos los tres artículos sobre Paleolítico podemos entender la importancia de la cita extranjera, fundamentalmente en francés. En el mismo sentido, seis de los nueve artículos del volumen de Cuadernos de Prehistoria y Arqueología Castellonense son de estudios clásicos o consideran el registro arqueológico de otros países. Por el contrario, Zephyrus con 34 artículos, dos sobre temas extrapeninsulares, siete sobre estudios clásicos - cuatro de ellos sobre epigrafía - y 11 sobre arte - desde el Paleolítico a época medieval- tiene unos valores de citas extranjeras inesperados.

Sin embargo, para interpretar globalmente el manejo de la cita extranjera es necesario contar con una serie más amplia de cada revista.

\subsection{Las citas a publicaciones españolas}

La importancia relativa de las diversas publicaciones en la muestra queda reflejada en el cuadro 4. Las «Revistas y Series monográficas» y las «Monografías», por su peso en la muestra y por la antigüedad de sus citas, se estudian por décadas durante los siglos XIX y XX (Fig. 1). El diferente comportamiento de los dos tipos de documentos está en relación con las pautas de difusión científica de cada época. Para saber si las citas anteriores a las primeras (cinco del siglo XVIII) y a las segundas (14 del siglo XVI, 22 del siglo XVII y 55 del siglo XVIII) son indicativas del desarrollo de la investigación arqueológica, sería necesario pasar de la cita al tipo de documento a la identificación de su título, ya que en las revistas figuran artículos de Arte e Historia Medieval y Mo-
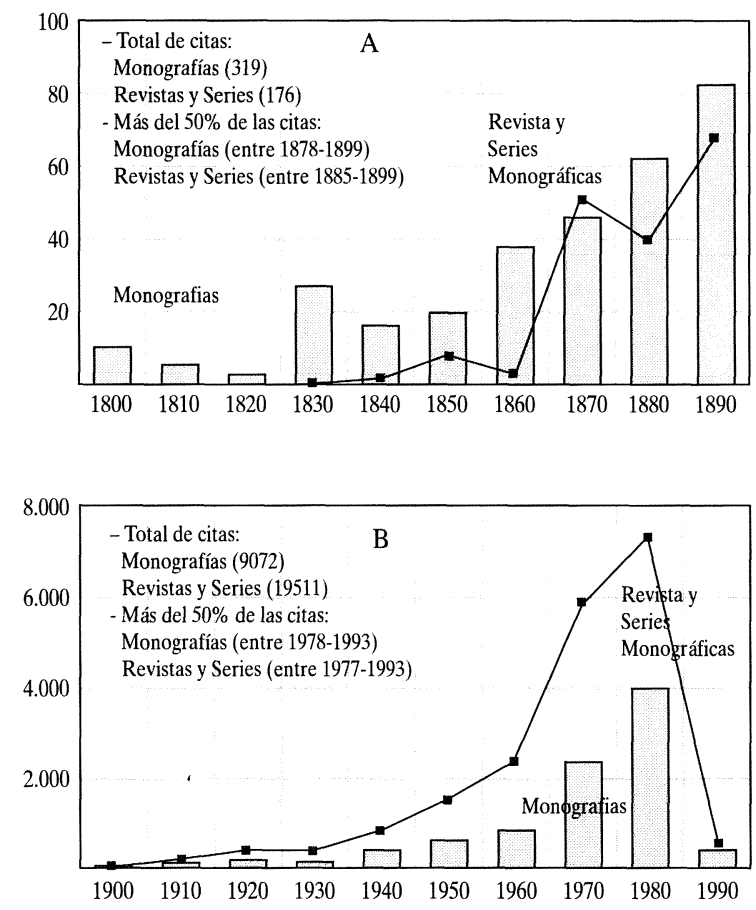

Fig. 1. Evolución por décadas de las Revistas y Series monográficas y Monografías en las revistas de la muestra: A. siglo XIX y B. siglo XX.

derna. En cualquier caso, algunas de las citas más antiguas se relacionan con el anticuarismo del que arranca una parte de la Arqueología. Otras se corresponden con el manejo de documentos históricos con información local y regional, como el catastro del Marqués de la Ensenada, habitual en los últimos años desde la Arqueología Espacial y del Paisaje.

\begin{tabular}{|l|r|r|r|r|c|c|}
\hline & Inicio & Final & Total citas & $\%$ citas & Total autocitas & \% Autocitas \\
\hline $\mathrm{Ar}$ & 1771 & 1995 & 19.842 & 59,27 & 2.107 & 10,61 \\
\hline $\mathrm{Mo}$ & 1513 & 1993 & 9.501 & 28,38 & 671 & 7,06 \\
\hline $\mathrm{Co}$ & 1908 & 1993 & 3.268 & 9,76 & 551 & 16,86 \\
\hline $\mathrm{Te}$ & 1955 & 1994 & 380 & 1,13 & 121 & 31,84 \\
\hline $\mathrm{ML}$ & 1959 & 1994 & 252 & 0,75 & 106 & 42,06 \\
\hline $\mathrm{In}$ & 1912 & 1992 & 42 & 0,12 & 11 & 26,19 \\
\hline Ot & 1639 & 1993 & 191 & 0,57 & 22 & 11,51 \\
\hline Total & & & 33.476 & & 3.589 & 10,72 \\
\hline
\end{tabular}

Cuadro 4. Período cubierto por cada tipo de documento, importancia relativa de los mismos y su relación con las autocitas de autor.

T. P., 53, n. ${ }^{\circ} 1,1996$ 


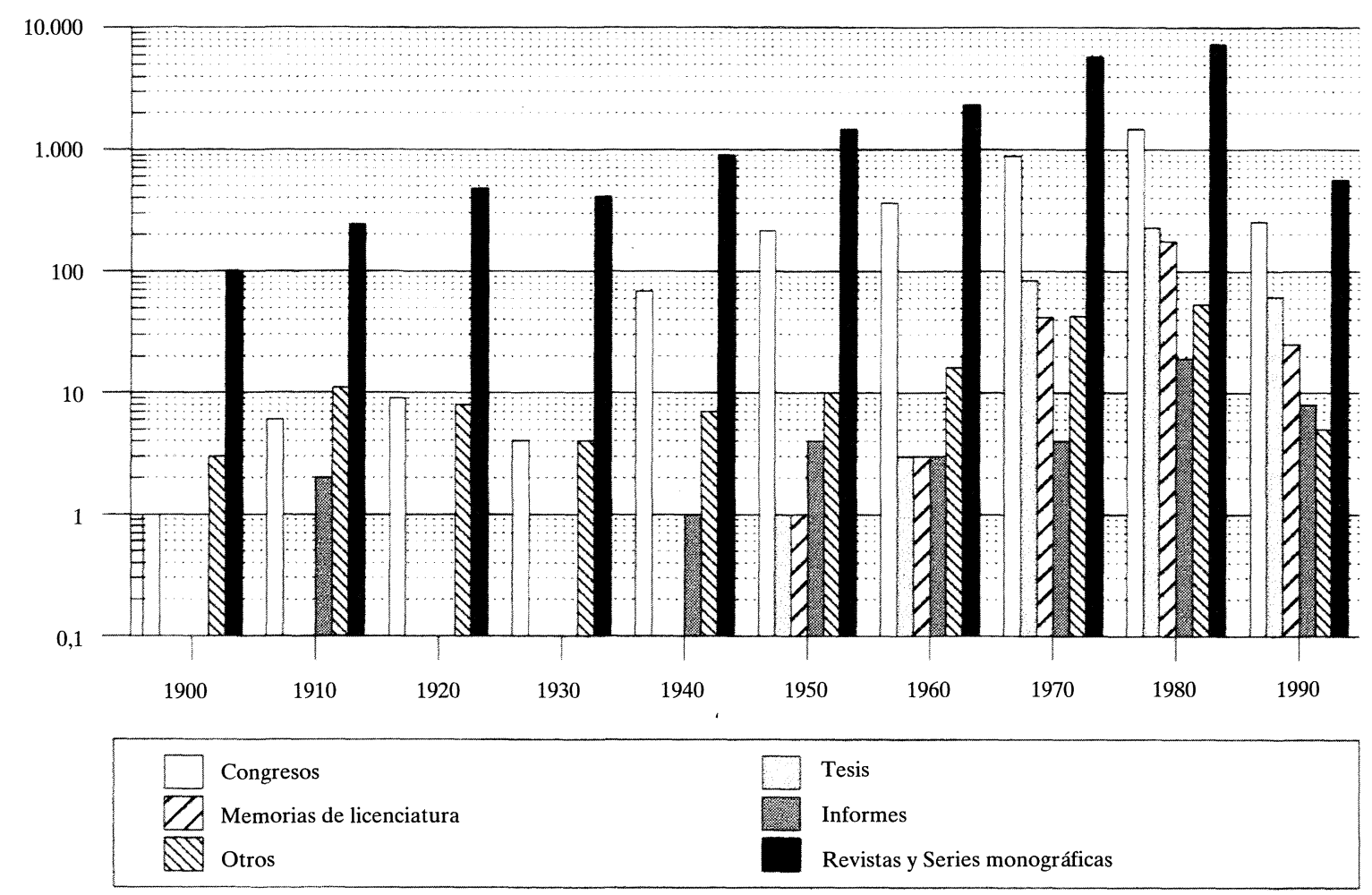

Fig. 2. Evolución de los tipos de documentos durante el siglo XX en las revistas de la muestra.

Durante las cuatro primeras décadas del siglo XIX, a pesar de las alternancias en las citas que relacionamos con la inestabilidad política, los valores son siempre crecientes. Este desarrollo de las monografías sobre Arqueología coincide con la aparición de citas a revistas en la década de los 30 . Sin embargo, el volumen de citas por décadas a ambos tipos de documentos es diferente. Las «Monografías» tienen un crecimiento sostenido durante todo el siglo mientras las citas a «Revistas y Series monográficas» sufren altibajos, si bien pasan de una cita en la década de 1830 a 67 en la de 1890 . Este papel preponderante de las primeras respecto a las segundas en el siglo XIX refleja bien los primeros tanteos de institucionalización de la Arqueología.

El rasgo más llamativo de la evolución de las citas durante el siglo XX es que, desde su inicio, las recibidas por «Revistas y Series monográficas» superan a las correspondientes a «Monografías». Así, las primeras pasan de 100 en la primera década a 7.294 en la de
1980 (3), mientras las valores de las citas a «Monografías» para ese mismo periodo oscilan entre 77 y 4.028. En general, hasta los años 50 las citas se duplican prácticamente de una fase a la siguiente con la única excepción de la Guerra Civil. La década de los 70 supone un cambio en el volumen de citas, ya que más de la mitad del total se concentra desde entonces. La importancia de este período en el conjunto de la muestra expresa el enorme desarrollo de la investigación arqueológica durante el mismo, ya señalado para las revistas de Arqueología medieval (Salvatierra, 1990: 19-27) y paralelo al que se constata en otros campos de las Ciencias Sociales y $\mathrm{Hu}-$ manas (Sánchez Nistal, 1995: 560).

Los «Congresos», «Informes» $\mathrm{y}$ «Otros» están presentes a lo largo del siglo XX (Fig. 2). La mayor proporción de citas de los primeros

(3) A este respecto no valoramos los datos de la presente década por estar infrarrepresentada en la cita por falta de perspectiva temporal. 
se debe a que, generalmente, son los únicos publicados. «Tesis» y «Memorias de licenciatura» aparecen a mediados de los 50 , y cada vez más desde entonces, en relación con la consolidación de la enseñanza universitaria y las disposiciones legales correspondientes (Ruiz-Zapatero, 1993: 48). Tratándose de documentos inéditos, los altos valores de autocitas de autor (Cuadro 4) sugieren una utilización restringida fundamentalmente a los equipos de investigación donde se generan posiblemente por la inadecuada difusión de este tipo de documentos.

\section{AUTORÍA}

La mayor parte de los artículos publicados está firmada por un solo autor. El valor medio de la coautoría, calculado a partir de la relación número de firmas/número de trabajos $(\mathrm{F} * \mathrm{~N} / \mathrm{N})$, es de 1,74 (Fig. 3A). Cuadernos de Prehistoria de la Universidad de Granada y Cypsela destacan y, especialmente, la primera donde los artículos de tres o más autores están igualmente representados (por encima del 10\%).

En cuanto a su evolución durante el período estudiado se observa un crecimiento de los artículos firmados por más de cuatro autores (5\%), y una relación complementaria entre los artículos publicados por uno y dos autores hasta 1990 (Fig. 3C). Desde entonces el decrecimiento de los firmados por un autor se complementa con un ascenso de los firmados por más de tres. Esta clara tendencia al incremento de la coautoría, aunque no supera la media de dos autores (Fig. 3B), sigue la tónica general de las Humanidades y Ciencias Sociales (Sánchez Nistal, 1995: 571-572).

La relación $\mathrm{n}^{\circ}$ pags. $/ \mathrm{n}^{\circ}$ arts. $(\mathrm{P} / \mathrm{A})$ es un indicador adicional de la importancia de la publicación individual. Sólo un tercio de la muestra se sitúa por debajo del valor medio ponderado de P/A $(21,6)$ (Fig. 4 y Cuadro 1). Algunas consecuencias derivadas de la tendencia a publicar artículos cuya longitud se aproxima más a la de las monografías, y de la irregularidad en la aparición de las revistas, se comentan en el apartado 6.

Las ventajas de la colaboración en la publicación son un tema discutido. La transferencia

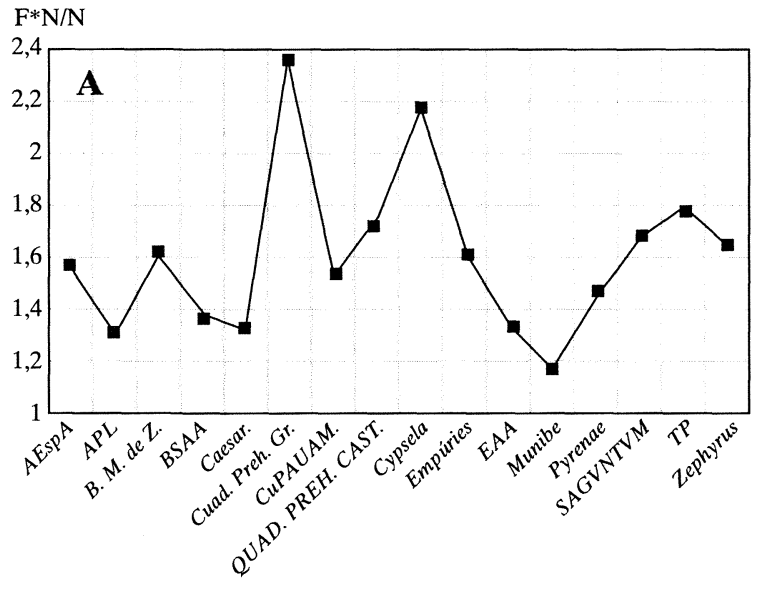

Revistas
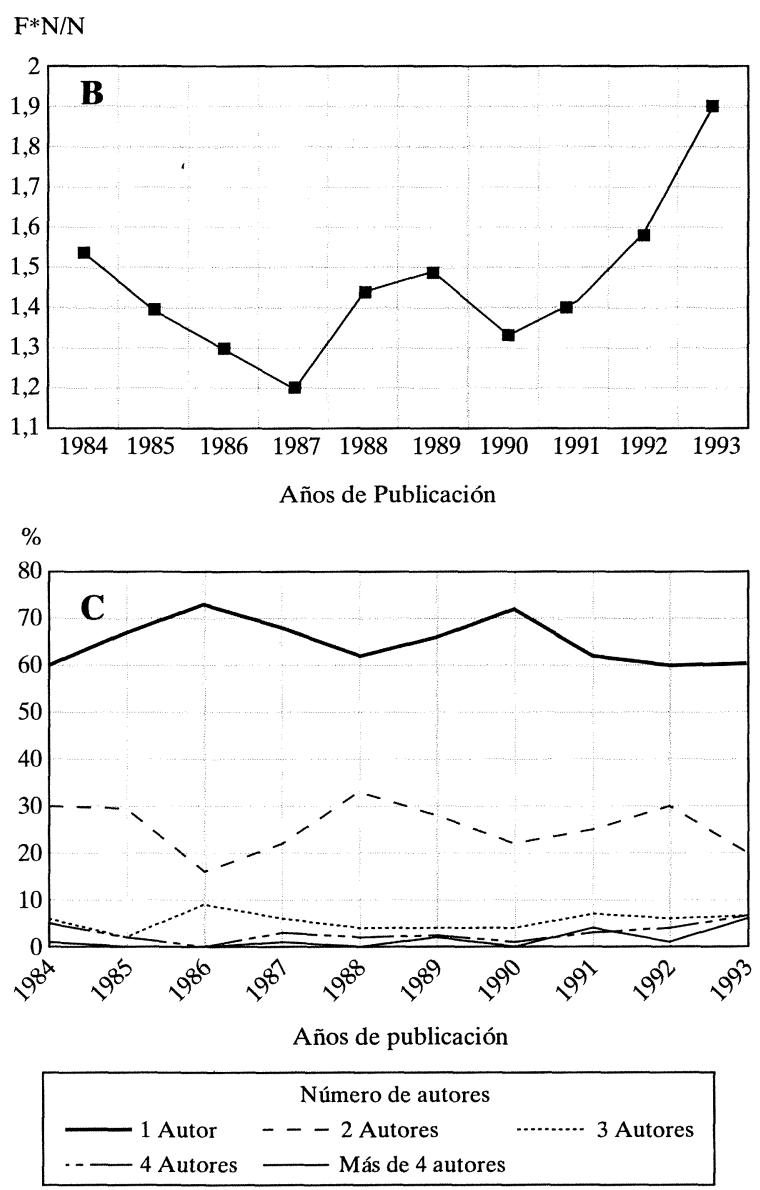

Fig. 3. Estudio de la coautoría a partir de la relación número de firmas/trabajos (F*N/N). A: Por revistas. 1. AEspA; 2. APL; 3. Boletín. Museo de Zaragoza; 4. BSAA; 5. Caesaraugusta; 6. Cuad.Preh.Gr.; 7. CuPAUAM; 8. QUAD.PREH.CAST.; 9. Cypsela; 10. Empúries; 11. EAA; 12. Munibe; 13. Pyrenae; 14. SAGVNTVM; 15. $T P ; 16$. Zephyrus. B: Por años de publicación. C: Por años de publicación y desglosada por autores. 


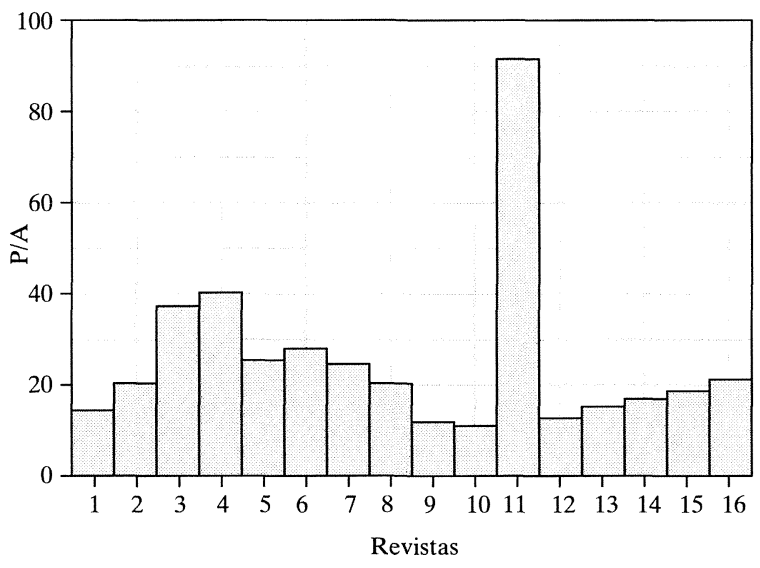

Fig. 4. Relación número de páginas por artículo (P/A) en las revistas de la muestra.

1. AEspA; 2. APL; 3. Boletín. Museo de Zaragoza; 4. BSAA; 5. Caesaraugusta; 6. Cuad.Preh.Gr.; 7. CuPAUAM; 8. QUAD.PREH.CAST.; 9. Cypsela; 10. Empúries; 11. EAA; 12. Munibe; 13. Pyrenae; 14. SAGVNTVM; 15. TP; 16. Zephyrus.

de información es independiente del número de firmantes. Sin embargo, en todos los campos científicos, salvo quizá en Humanidades, cada vez más el aumento del número de firmantes se correlaciona con la calidad del artículo y, por lo tanto, con su impacto (Ferreiro, 1993: 76-77; Villagrá, 1992: 12-13). La significación que sigue teniendo la publicación individual en Prehistoria y Arqueología (Rovira, 1994: 58) sugiere que la especialización, la multidisciplinariedad y la práctica del trabajo en equipo (Rodríguez et alii, 1993: 24 y 36), no han calado todavía suficientemente en estas disciplinas.

\section{5. ÁMBITO TERRITORIAL}

La gran cantidad de publicaciones sobre Prehistoria y Arqueología identificadas desde las citas de las revistas de la muestra aconsejó manejar la Comunidad Autónoma como unidad espacial de análisis. Este marco es meramente convencional. Los resultados de nuestro estudio muestran que, en unos casos, explica algunos aspectos del panorama editorial mientras que en otros lo realmente significativo es la propia revista, que comparte su orientación temática específica con revistas de otras Comunidades. Esto debe tenerse en cuenta cuando se vean clasificadas de forma global las publicaciones como "catalanas", "castellano-leonesas", etc.

El desarrollo regional de la investigación arqueológica se ha estudiado a partir del lugar de publicación con una doble estrategia. Primero, se ha definido la distribución territorial de las revistas distinguiendo el papel de las capitales de provincia en la edición. Después, se ha reducido la muestra a aquellas publicaciones con un mínimo de 10 citas para valorar de dos maneras las interrelaciones entre los investigadores que publican en ellas: a) desde las citas que reciben las Comunidades Autónomas; y b) extrayendo las publicaciones citadas por un máximo de dos revistas. El lugar de edición no basta como indicador de la temática de una publicación. Para soslayar esta limitación se han manejado, junto a una muestra representativa, otros datos como los territorios a los que se refieren los artículos de las revistas de la muestra, excepto la serie
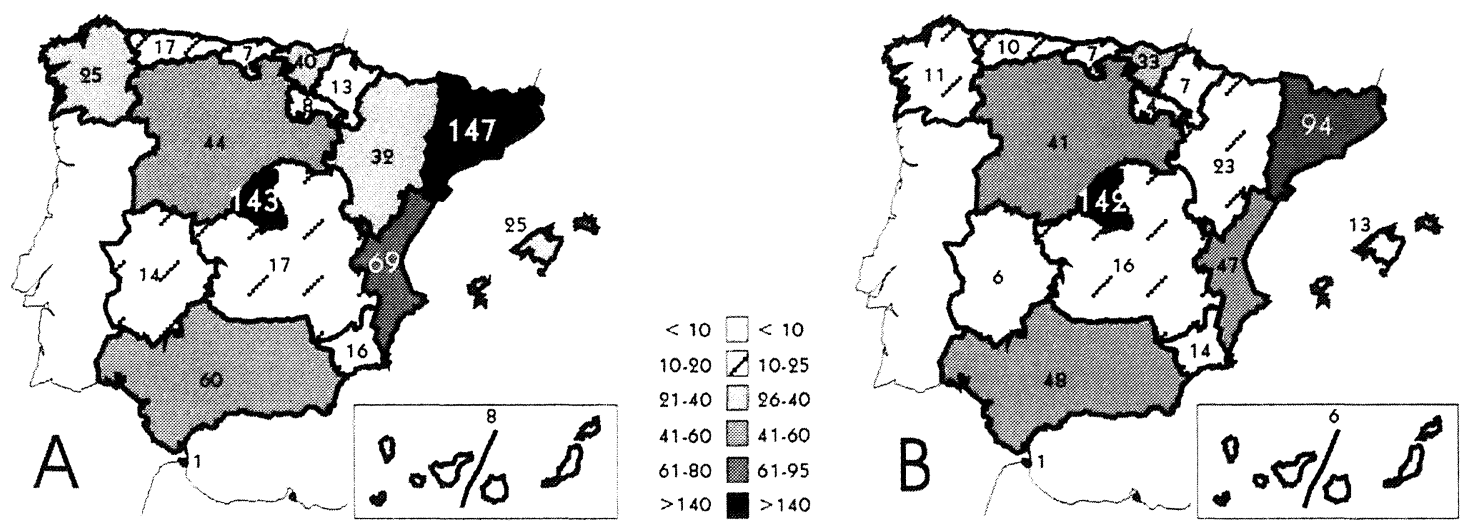

Fig. 5. Clasificación total de revistas: A: Número de publicaciones por Comunidad Autónoma. B: Número de publicaciones en capitales de provincia por Comunidad Autónoma.

T. P., 53, n. ${ }^{\circ} 1,1996$ 


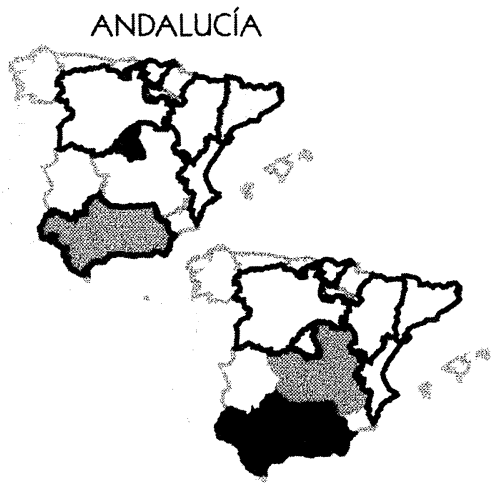

Cuad. Preh. Gr.

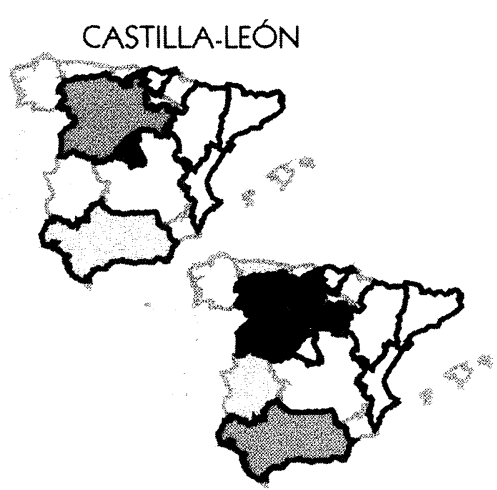

BSAA, Zephyrus

PAÍS VASCO

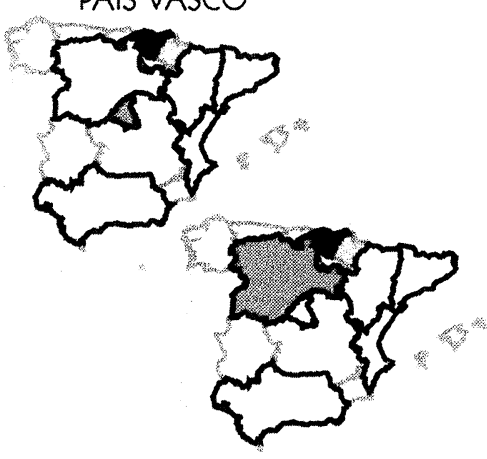

ARAGON

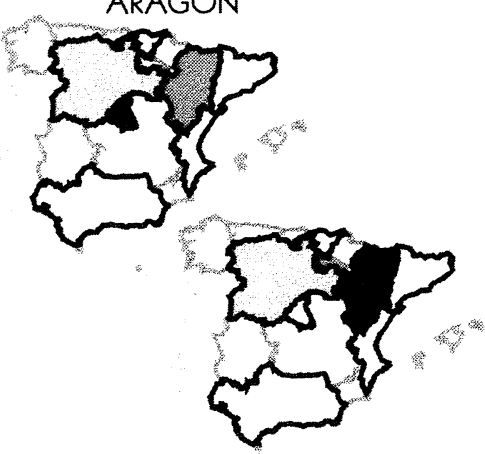

Bol. Mus. Zaragoza, Caesaraugusta

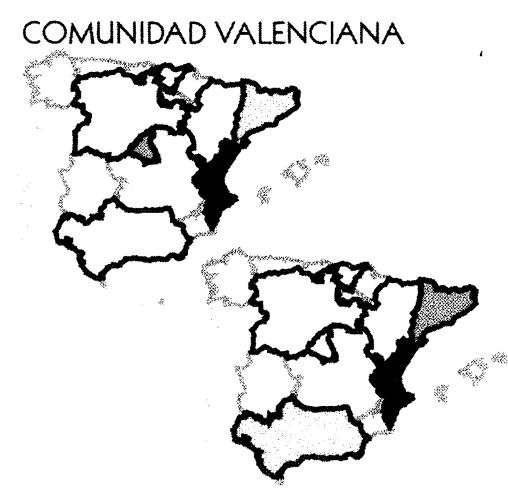

APL, QUAD. PREH. CAST., SAGVNTVM SINTTESIS

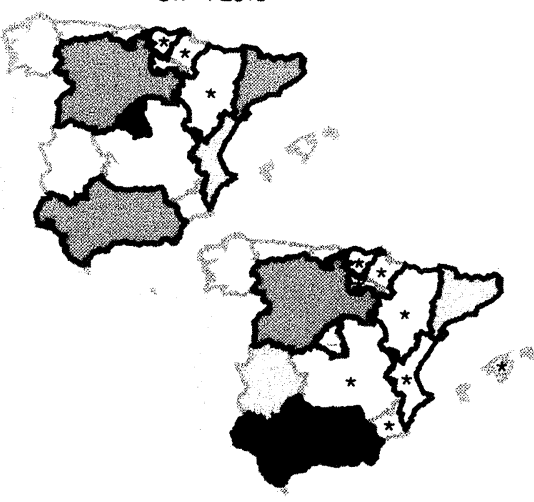

CATALUINA

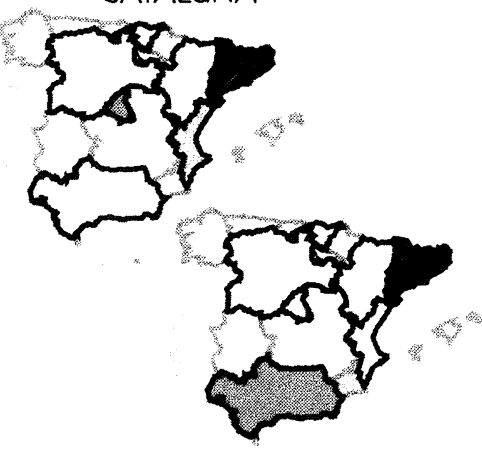

Cypsela, Empúries, Pyrenae

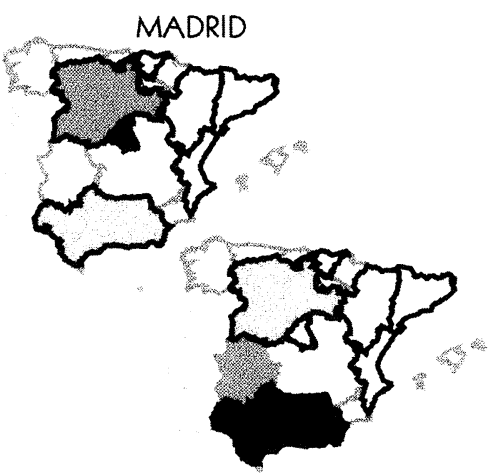

AEspA, CuPAUAM, EAE, TP

Primera $\square$ Segunda $\square$ Tercera

Comunidades Autónomas con revistas

Comunidades Autónomas con una aparición en los mapas * de citas o artículos

EAA, Munibe

Fig. 6. Las tres Comunidades Autónomas más citadas a partir de los datos del anexo (mapa superior) y de los artículos en las revistas de la muestra (mapa inferior). Cuando varias Comunidades tienen el mismo valor se representan todas. Los mapas sintéticos están a mayor tamaño. 


\begin{tabular}{|l|r|r|r|r|r|r|r|}
\hline & And. & Ara. & C.-L. & Cat. & C.Va. & Mad. & P.Vas. \\
\hline Andalucía (And.) & 47 & 1 & 23 & 14 & 9 & 42 & \\
\hline Aragón (Ara.) & & 37 & 5 & 7 & 5 & 4 & \\
\hline Asturias & & & 10 & 1 & 1 & 5 & \\
\hline Baleares & & & & 9 & 9 & 8 & \\
\hline Canarias & & & 3 & & & & \\
\hline Cantabria & & & 7 & 1 & & 3 & \\
\hline Castilla-León (C.-L.) & 1 & 3 & 57 & 4 & 1 & 12 & 2 \\
\hline Castilla-La Mancha & & 2 & 7 & 2 & 3 & 7 & \\
\hline Cataluña (Cat.) & & 2 & 8 & 225 & 12 & 13 & \\
\hline C. Valenciana (C.Va.) & & & 5 & 8 & 144 & 7 & \\
\hline Extremadura & & & 10 & 2 & 3 & 16 & \\
\hline Galicia & & & 5 & & & 4 & \\
\hline La Rioja & & 4 & 1 & & & & \\
\hline Madrid (Mad.) & & & 1 & & & 6 & \\
\hline Navarra & & 1 & 6 & & & & 1 \\
\hline País Vasco (P.Vas.) & & 1 & 5 & 1 & & 2 & 34 \\
\hline Región de Murcia & & 3 & 4 & 12 & 3 & 13 & \\
\hline Varias Comunidades & & 1 & 31 & 21 & 5 & 30 & \\
\hline Extranjero & 1 & 5 & 8 & 38 & 6 & 18 & \\
\hline Total & 49 & 60 & 196 & 345 & 201 & 190 & 37 \\
\hline
\end{tabular}

Cuadro 5. Distribución territorial por Comunidades Autónomas de las citas desde las publicaciones con más de 10 citas. Se incluye la serie Excavaciones Arqueológicas en España.

Excavaciones Arqueológicas en España. Igualmente, la cita extranjera (apartado 3.1) por sí sola no es un buen marcador de la posible ampliación del ámbito territorial aunque, en ocasiones, puede reforzar los resultados obtenidos con las estrategias anteriores.

En cuanto al primer objetivo (Fig. 5), para valorar la vigencia temporal de los datos se han comparado las publicaciones que reciben más de 100 citas en el total de la muestra (Anexo), las cuales representan el 63\%, con las de los años 1986, 1990, 1992 y 1993. El resultado confirma la representatividad de esa vigencia: 18 publicaciones aparecen todos esos años, cuatro en tres, cuatro en dos y cuatro sólo en uno.

Las Comunidades Autónomas que tienen más publicaciones son Cataluña y Madrid (Fig. 5A), la primera por el desarrollo de la investi- gación arqueológica en las cuatro provincias catalanas desde principios de siglo y la segunda por la concentración en la capital de las instituciones estatales de investigación y de gestión de la arqueología junto a las Universidades con mayor peso. Además, la importancia de sus publicaciones no se puede explicar por el desarrollo de la investigación arqueológica en la propia región.

No existe una relación directa entre el territorio de la Comunidad Autónoma y las publicaciones que editan. Sirva como ejemplo la semejanza en el número de revistas de Castilla-La Mancha y la Región de Murcia.

Las publicaciones se concentran en las capitales de provincia (Fig. 5B), sedes de los centros de investigación y los organismos de gestión. La excepción la constituyen Cataluña, la Comunidad Valenciana y Baleares donde la comarcalización es muy marcada: una media del $39 \%$ de las publicaciones se edita fuera de las capitales.

En cuanto a la segunda estrategia, se han establecido las tres Comunidades más citadas en cada caso (Fig. 6 mapa superior y Cuadro 5). Madrid es la única siempre citada. Además, recibe el mayor número de citas en todos los casos menos desde Cataluña, Comunidad Valenciana y País Vasco, donde ocupa el segundo lugar tras la respectiva Comunidad Autónoma. En las dos primeras, en el tercer lugar está la Comunidad de su ámbito lingüístico y en el País Vasco, Navarra. En las restantes el segundo lugar se corresponde con la propia Comunidad. Otras se citan por tres de ellas (Andalucía,

\begin{tabular}{|l|l|l|}
\hline I. Más de 10 CC.AA. & II. Entre 10 y 6 CC. AA. & III. Menos de 6CC. AA. \\
\hline$-T P$ & - Cuad.Preh.Gr. & - Cypsela \\
- Zephyrus & - Caesaraugusta & - Empúries \\
& - BSAA & - Pyrenae \\
& - APL & - QUAD. PREH. CAST. \\
& - SAGVNTVM & - EAA \\
& - AEspA & \\
& - CuPAUAM & \\
& - EAE & \\
& - Munibe & \\
\hline
\end{tabular}

Cuadro 6. Número de Comunidades Autónomas citadas desde las revistas de la muestra. Se incluye la serie Excavaciones Arqueológicas en España. 
Castilla-León y Cataluña) y la Comunidad Valenciana por dos. Hay Comunidades que no se citan. Ahora bien, esto no significa necesariamente que las citas a una Comunidad Autónoma desde una revista concreta de fuera de ella expresen un interés por esa comunidad (véase más abajo Cuadernos de Prehistoria de la Universidad de Granada).

Para evaluar esta última cuestión, se han clasificado las revistas de la muestra (salvo el Boletín. Museo de Zaragoza) en tres categorías según el número de Comunidades citadas y en orden creciente (Cuadro 6). En el grupo III se sitúan las revistas de las Comunidades Autónomas donde la comarcalización es más fuerte: todas las catalanas, una de la Comunidad Valenciana y una vasca. Por ejemplo, Cypsela sólo cita revistas catalanas y madrileñas $(5 \%)$. En sus normas de publicación (volumen X, 1993: 210) señala como ámbito territorial preferente «las comarcas gerundenses, Cataluña y regiones adyacentes». Ello no excluye que en algún volumen dicho ámbito se amplíe por la temática de los artículos (apartado 3.1).

Esa revista es un ejemplo extremo de una tendencia general de las publicaciones arqueológicas en España: entre las revistas citadas la intensidad de citas decrece con la distancia a la región de publicación. En este contextơ, la cita a revistas de fuera de la región se debería a que publican trabajos referidos a ella. Esa tendencia se expresa también en la importancia relativa de las citas a la propia Comunidad Autónoma de cada revista (Cuadro 7). Las revistas madrileñas, donde los valores de esas citas no llegan al $5 \%$, serán objeto de un comentario

\begin{tabular}{|l|l|l|}
\hline 1. Más del $75 \%$ & 2. Entre el 50 y el $75 \%$ & 3. Entre el 49 y el $25 \%$ \\
\hline - Cypsela & - Cuad.Preh. Gr. & - Caesaraugusta \\
- BSAA & - Empúries & - Zephyrus \\
- QUAD.PREH.CAST. & - Pyrenae & \\
& - APL & \\
& - SAGVNTVM & \\
& - EAA & \\
& - Munibe & \\
\hline
\end{tabular}

Cuadro 7. Citas en orden creciente a la propia Comunidad Autónoma desde las revistas de la muestra. Se han excluido las revistas madrileñas. aparte. Sólo Caesaraugusta (27\%) y Zephyrus $(38 \%)$ tienen un carácter general.

El análisis de los artículos permite contrastar esa hipótesis destacando los referidos a varias Comunidades y también a las tres que merecen una atención preferente (Fig. 6 mapa inferior y Cuadro 8). Los sumarios no suelen ser suficientemente informativos ya que no siempre reflejan fielmente los títulos ni la referencia temática, espacial y cronológica, al contrario de lo que suele suceder en las revistas de Ciencia y Tecnología.

\begin{tabular}{|l|r|r|r|r|r|r|r|}
\hline & And. & Ara. & C.-L. & Cat. & C.Va. & Mad. & P.Vas. \\
\hline Andalucía (And.) & 56 & 1 & 23 & 14 & 9 & 42 & 1 \\
\hline Aragón (Ara.) & & 35 & 5 & 7 & 5 & 4 & 1 \\
\hline Asturias & & & 10 & 1 & 1 & 5 & \\
\hline Baleares & & & & 9 & 9 & 8 & \\
\hline Canarias & & & 3 & & & & \\
\hline Cantabria & & & 7 & 1 & & 3 & \\
\hline Castilla-León (C.-L.) & & 5 & 84 & 4 & 2 & 14 & 3 \\
\hline Castilla-La Mancha & 1 & 3 & 7 & 2 & 3 & 5 & \\
\hline Cataluña (Cat.) & & 2 & 8 & 222 & 12 & 13 & \\
\hline C. Valenciana (C.Va.) & & & 5 & 8 & 144 & 7 & \\
\hline Extremadura & & & 10 & 2 & 3 & 16 & \\
\hline Galicia & & & 5 & & & 4 & 1 \\
\hline La Rioja & & 4 & 1 & & & & \\
\hline Madrid (Mad.) & & & 1 & & & 6 & \\
\hline Navarra & & 1 & 6 & & & & 3 \\
\hline País Vasco (P.Vas.) & & 1 & 5 & 1 & & 2 & 79 \\
\hline Región de Murcia & & 3 & 4 & 12 & 3 & 13 & \\
\hline Varias Comunidades & 2 & 1 & 31 & 21 & 5 & 30 & 8 \\
\hline Extranjero & 1 & 5 & 8 & 38 & 6 & 18 & 16 \\
\hline Total & 60 & 61 & 223 & 342 & 202 & 190 & 112 \\
\hline
\end{tabular}

Cuadro 8. Comunidades Autónomas estudiadas en los artículos de las revistas de la muestra.

Para interpretar los resultados bibliométricos es necesaria su progresiva contextualización desde el ámbito general, en nuestro caso la Comunidad Autónoma, al más restringido de la revista. Si las publicaciones reflejan un notable localismo, su explicación en cada Comunidad Autónoma requiere un estudio específico ya que el desarrollo de la investigación arqueológica no 
es uniforme en todo el Estado, ni en infraestructura, ni en la atención a los distintos períodos históricos (Fig. 7A) ni a la temática (Fig. 7B).
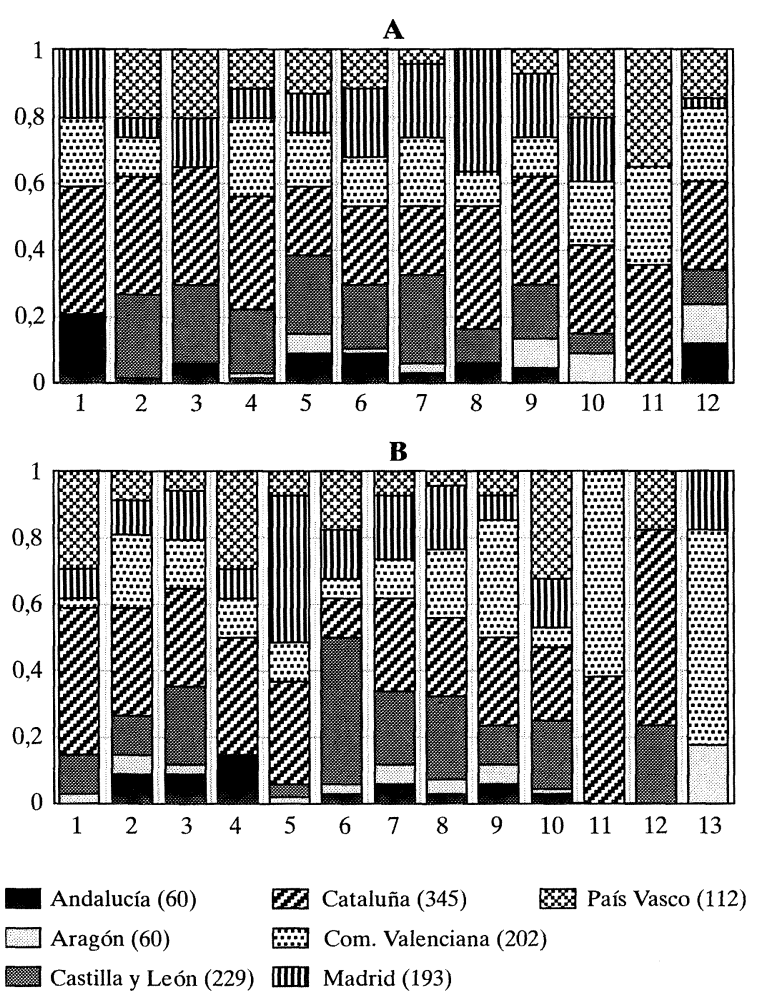

Fig. 7. A Períodos tratados en los artículos de las revistas de la muestra por Comunidades Autónomas (entre paréntesis los totales): 1 Origen del Hombre. 2 Paleolítico. 3 Epipaleolítico. 4 Neolítico. 5 Calcolítico. 6 Edad del Bronce. 7 Edad del Hierro/Ibérico. 8 Colonizaciones. 9 Romano/Paleocristiano. 10 Medieval. 11 Moderna/Contemporánea. 12 Indeterminado. B: Temática por Comunidades Autónomas en los artículos de las revistas de la muestra (entre paréntesis los totales). 1 Estudios del Medio. 2 Asentamientos. 3 Enterramientos. 4 Antropología Física. 5 Economía/Sociedad. 6 Religión/Culto. 7 Industrias/Utiles. 8 Arte. 9 Epigrafía/Numismática. 10 Síntesis. 11 Pecios. 12 Datación. 13 Indeterminado.

Los estudios interregionales y sobre el extranjero se aprecian en el cuadro 8. Las revistas de todas las Comunidades Autónomas, salvo las aragonesas y de la Comunidad Valenciana, contienen artículos cuyo ámbito territorial ignora los límites administrativos actuales. Son destacables especialmente en las revistas de Castilla-León, Madrid y Cataluña. Considerando además los artículos sobre materiales o yaci-

\begin{tabular}{|l|l|l|l|l|l|l|}
\hline & APL & Q.P.C. & SAGVNTVM & Cypsela & Empúries & Pyrenae \\
\hline 1 & 26 & & & & & \\
\hline 2 & 25 & & & & & \\
\hline 3 & 23 & & & & & \\
\hline 4 & 18 & & & & & \\
\hline 5 & 14 & 22 & & & & \\
\hline 6 & 13 & & & & & \\
\hline 7 & 12 & & & & & \\
\hline 8 & 11 & & & & & \\
\hline 9 & 10 & & & & & \\
\hline 10 & 10 & & & & & \\
\hline 11 & & & 11 & & & \\
\hline 12 & & & & & 12 & \\
\hline 13 & & & & 43 & & \\
\hline 14 & & & & 40 & 13 & \\
\hline 15 & & & & 26 & & \\
\hline 16 & & & & 23 & & \\
\hline 17 & & & & 17 & 14 & \\
\hline 18 & & & & 16 & 14 & \\
\hline 19 & & & & 15 & 47 & \\
\hline 20 & & & & 14 & & \\
\hline 21 & & & & 13 & 11 & \\
\hline 22 & & & & 11 & & \\
\hline 23 & & & & & 22 & \\
\hline 24 & & & & & & 13 \\
\hline 25 & & & & & & 12 \\
\hline 26 & & & & & & 11 \\
\hline 27 & & & & & & 10 \\
\hline 28 & & & & & & 10 \\
\hline
\end{tabular}

Cuadro 9. Publicaciones citadas exclusivamente por una o dos de las revistas catalanas y de la Comunidad Valenciana de la muestra (Q.P.C. es $Q U A D$. PREH.CAST.): 1 La Labor del SIP y su Museo, Valencia. 2 Serie Arqueológica. Universidad de Valencia. 3 Memorias de la Sociedad Arqueológica Valenciana. 4 Emerita. Madrid. 5 Boletín de la Sociedad Castellonense de Cultura. 6 Saitabi, Valencia. 7 Revista del Instituto de Estudios Alicantinos. 8 Munibe. San Sebastián. 9 Anales del Centro de Cultura Valenciana. 10 Al-Qantara. Madrid. 11 Extremadura Arqueológica, Mérida. 12 Butlletí Arqueologic, Tarragona. 13 Serie Monográfica. Centre d'Investigacions Arqueológiques, Girona. 14 Annals de l'Institut d'Estudis Gironins. 15 Estudis sobre Temes del Baix Empordá, Girona. 16 Quaderns del Centre d'Estudis Comarcals de Banyoles, Barcelona. 17 Monografies Emporitanes, Barcelona. 18 Excavacions Arqueológiques a Catalunya, Barcelona. 19 Informació Arqueológica, Barcelona. 20 Memories d'Excavació. TED'A, Tarragona. 21 Cota Zero, Barcelona. 22 Vitrina, Olot. 23 Ilerda, Lleida. 24 Excavaciones Arqueológicas en Asturias. 25 Programa de Investigaciones Protohistóricas, Barcelona. 26 Boletín de la Real Sociedad Arqueológica Tarraconense. 27 Publicaciones Eventuales. Instituto de Arqueología y Prehistoria, Barcelona. 28 Arrahona, Sabadell.

T. P., 53, n. ${ }^{\circ} 1,1996$ 
mientos extranjeros, sobresalen las catalanas, madrileñas y vascas.

Sin embargo, como puede verse, la inmensa mayoría de los artículos aborda una temática que tiene en cuenta dichos límites. La comparación de los mapas de la figura 6 y de sus datos fuente (Cuadros 6 y 8) ayuda a comprender la dinámica de las conexiones regionales preferentes en tanto que los cuadros 9, 10 y 11 expresan el énfasis sobre la propia región.

Si las revistas de Madrid no reflejan la investigación regional sino la del conjunto de España, las citas a las publicaciones de las demás Comunidades Autónomas serían indicativas del desarrollo de la investigación arqueológica en cada una de ellas. Excluyendo las autocitas, todas las revistas madrileñas de la muestra citan, entre las tres primeras, a revistas de Castilla-León y Cataluña, dos de la Comunidad Valenciana y las otras dos de Andalucía.

La referencia a la Comunidad de Madrid desaparece desde los artículos confirmando el carácter subsidiario que tiene la investigación arqueológica en la propia región para explicar la cita a las revistas madrileñas. La primera posición que ocupaba en citas es sustituida por la propia Comunidad de Andalucía, Aragón y CastillaLeón en artículos. Donde figuraba en segundo lugar se encuentran ahora otras Comunidades: en Cataluña, Andalucía; en la Comunidad Valenciana, Cataluña y en el País Vasco, Castilla-León.

Cuadernos de Prehistoria de la Universidad de Granada cita revistas catalanas, las cuales no citan revistas andaluzas. Empúries y Pyrenae publican artículos, tanto de investigadores catalanes como andaluces, sobre Andalucía. En cambio, en Cuadernos de Prehistoria de la Universidad de Granada no hay artículos sobre Cataluña. De hecho, todos se ocupan de Andalucía salvo uno relativo a Castilla-La Mancha, área vinculada a la investigación de la Universidad de Granada, otro sobre el extranjero y dos referidos a varias Comunidades Autónomas. El hecho de que la revista granadina se encuentre en el grupo II del cuadro 6 pone de manifiesto la necesidad de combinar los datos de citas y de temática de los artículos para conocer adecuadamente las conexiones interterritoriales.

La atención que muestra Cuadernos de Prehistoria de la Universidad de Granada por revistas de otras Comunidades se puede relacionar con el hecho de que Andalucía es una zona clásica de la investigación arqueológica española desde el Calcolítico hasta la época romana (Rodríguez et alii, 1993: 21). Esto se refleja en la presencia de artículos sobre esta Comunidad en las revistas de todas las restantes y en el papel destacado que tiene en las más generales como las madrileñas y las castellano-le. onesas (Cuadro 6, grupos I y II).

Tanto Boletín del Seminario de Estudios de Arte y Arqueología y Zephyrus, como las revistas madrileñas manifiestan una orientación de la investigación hacia la mitad occidental peninsular. Sólo las primeras dedican un número significativo de artículos a Cantabria, indicativos del interés de la Universidad de Valladolid $\mathrm{y}$, sobre todo, de Salamanca por la investigación sobre el Paleolítico (Fig. 7A).

El peso correspondiente a los artículos sobre Extremadura en las revistas castellanoleonesas y madrileñas puede explicarse por los estudios sobre Edad del Hierro y Colonizaciones reforzados, en las primeras, por los dedicados al Calcolítico. El hecho de que la lengua portuguesa (Cuadro 3) tenga en todas ellas, salvo Archivo Español de Arqueología, y muy especialmente en Boletín del Seminario de Estudios de Arte y Arqueología (18\%) y Cuadernos de Prehistoria y Arqueología de la Universidad Autónoma de Madrid (11,8\%) valores muy superiores a su media $(3,4 \%)$ en el conjunto de las citas extranjeras apoya esa orientación territorial.

No existe una conexión significativa entre los investigadores de las Comunidades vecinas catalana y aragonesa a juzgar por la baja intensidad tanto de las citas como de los artículos que respectivamente reciben. La segunda mantiene la importancia en artículos de la Comunidad castellano-leonesa comentada en relación con las citas. Sólo estas últimas y las aragonesas abordan temas riojanos.

Las revistas de la Comunidad Valenciana se ocupan preferentemente de tópicos tradicionales de la investigación arqueológica de la fachada mediterránea como las colonizaciones, la romanización y la neolitización (Fig. 7A). De ahí la importancia que, tanto por citas como por artículos, tienen Cataluña y Andalucía. 


\begin{tabular}{|l|c|c|c|c|}
\hline & B. M. de Z. & Caesaraugusta & EAA & Munibe \\
\hline 29 & 17 & 18 & & \\
\hline 30 & 11 & & & \\
\hline 31 & & 12 & & \\
\hline 32 & & & & 22 \\
\hline 33 & & & 25 & \\
\hline 34 & & & 20 & \\
\hline 35 & & & 19 & \\
\hline 36 & & & 10 & 47 \\
\hline 37 & & & 10 & \\
\hline 38 & & & & 73 \\
\hline 39 & & & & 47 \\
\hline 40 & & & & 30 \\
\hline 41 & & & & 20 \\
\hline 42 & & & & 17 \\
\hline 43 & & & & 14 \\
\hline
\end{tabular}

Cuadro 10. Publicaciones citadas exclusivamente por una o dos de las revistas aragonesas y vascas de la muestra: 29 Boletín. Museo de Zaragoza (B.M. de Z.). 30 Arqueología Aragonesa, Zaragoza. 31 Numisma, Madrid. 32 Veleia, Vitoria. 33 Boletín de la Institución Sancho el Sabio, Vitoria. 34 Estudios del Grupo Espeleológico Alavés, Vitoria. 35 Excavaciones en Navarra. 36 Arkeoikuska, Vitoria. 37 Investigaciones Arqueológicas en Álava, Vitoria. 38 Trabajos de Arqueología Navarra, Pamplona. 39 Kobie, Bilbao. 40 Cuadernos de Sección. Antropología/Etnografía, Prehistoria/ Arqueología, San Sebastián. 41 Cuadernos de Arqueología de Deusto. 42 Anuario de Eusko Folklore, San Sebastián. 43 Cuadernos de Etnología y Etnografía de Navarra, Pamplona.

La aparente ampliación del ámbito territorial de las revistas vascas se debe exclusivamente a los artículos sobre el Condado de Treviño, enclave castellano-leonés en Álava. La focalización de las publicaciones vascas se acompaña por la ausencia tanto de un volumen significativo de citas a las mismas desde las revistas de las restantes Comunidades Autónomas, salvo desde las de Madrid y Navarra, como de artículos sobre temas de arqueología vasca.

Como hemos visto, la línea editorial puede explicar la presencia e importancia relativa de las distintas regiones peninsulares. Sería necesario un estudio específico de cada revista para precisar la influencia de este factor en cada caso.

Concluimos este apartado comentando los cuadros 9 a 12 que expresan de forma muy clara la influencia del territorio y de la temática

\begin{tabular}{|c|c|c|c|c|c|c|}
\hline & $B S A A$ & Zephyrus & AEspA & CUPAUAM & EAE & $T P$ \\
\hline 4 & & 23 & & & & \\
\hline 10 & & & & 11 & & \\
\hline 11 & & & & 10 & & \\
\hline 31 & & & 14 & & & \\
\hline 35 & & 17 & & & & \\
\hline 38 & & 46 & & & & \\
\hline 41 & & & & & & 10 \\
\hline 44 & 18 & & & & & \\
\hline 45 & 11 & & & & & 12 \\
\hline 46 & 10 & & & & & 14 \\
\hline 47 & & & 13 & & & \\
\hline 48 & & & 12 & & & \\
\hline 49 & & 11 & & 14 & & \\
\hline 50 & & 12 & & 13 & & \\
\hline 51 & & 12 & & & 15 & \\
\hline 52 & & & & & & 29 \\
\hline 53 & & 37 & & & & 26 \\
\hline 54 & & 27 & & & & 22 \\
\hline 55 & & 23 & & & & 21 \\
\hline 56 & & & & & & 18 \\
\hline 57 & & 47 & & & & 16 \\
\hline 58 & & & & & & 15 \\
\hline 59 & & & & & & 15 \\
\hline 60 & & & & & & 14 \\
\hline 61 & & & & & & 11 \\
\hline 62 & & & & & & 11 \\
\hline 63 & & 15 & & & & 11 \\
\hline 64 & & & & & & 11 \\
\hline 65 & & & & & & 11 \\
\hline 66 & & 10 & & & & 11 \\
\hline 67 & & 33 & & & & 10 \\
\hline 68 & & & & & & 10 \\
\hline 69 & & 25 & & & & \\
\hline 70 & & 24 & & & & \\
\hline 71 & & 19 & & & & \\
\hline 72 & & 14 & & & & \\
\hline 73 & & 13 & & & & \\
\hline 74 & & 12 & & & & \\
\hline 75 & & 11 & & & & \\
\hline 76 & & 11 & & & & \\
\hline 77 & & 11 & & & & \\
\hline 78 & & 11 & & & & \\
\hline 79 & & 11 & & & & \\
\hline 80 & & 11 & & & & \\
\hline 81 & & 10 & & & & \\
\hline 82 & & 10 & & & & \\
\hline
\end{tabular}

Cuadro 11. Publicaciones citadas exclusivamente por una o dos de las publicaciones madrileñas y castellanoleonesas de la muestra: 4 Emerita, Madrid. 10 Al-Qantara, Madrid. 1 Extremadura Arqueoló- 
gica, Mérida. 31 Numisma, Madrid. 35 Excavaciones en Navarra. 38 Trabajos de Arqueología Navarra, Pamplona. 41 Cuadernos de Arqueología de Deusto. 44 Publicaciones de la Institución Tello Téllez de Meneses, Palencia. 45 Sautuola, Santander. 46 Estudios de Prehistoria y Arqueología Madrileñas. 47 Aula Orientalis, Sabadell. 48 Antigüedad y Cristianismo, Murcia. 49 Boletín Auriense, Ourense. 50 Revista de Estudios Extremeños, Badajoz. 51 Celtiberia, Soria. 52 Bolletí de la Societat Arqueológica Luliana, Palma de Mallorca. 53 Actas y Memorias de la Sociedad Española de Antropología, Etnografía y Prehistoria, Madrid. 54 Acta Arqueológica Hispánica, Madrid. 55 Boletín del Instituto de Estudios Asturianos, Oviedo. 56 Boletín de la Junta Superior de Excavaciones Arqueológicas, Madrid. 57 Memorias. Comisión de Investigaciones Paleontológicas y Prehistóricas, Madrid. 58 Anales de Prehistoria y Arqueología, Murcia. 59 Pontevedra Arqueológica. 60 Baetica, Málaga. 61 Revista de la Uni ersidad Complutense, Madrid. 62 Memorias de Historia Antigua, Oviedo. 63 Boletín del Instituto de Estudios Giennenses, Jaén. 64 Anuario de Prehistoria Madrileña. 65 Publicaciones del Patronato de las Cuevas Prehistóricas de la Provincia de Santander. 66 Arqueología. Ministerio de Cultura, Madrid. 67 Oretania, Linares. 68 Inventaria Archaeologica, Madrid. 69 Acta Salmanticensia, Salamanca. 70 Memorias del Seminario de Prehistoria y Arqueología, Salamanca. 71 Mainake, Málaga. 72 Gallaecia, Santiago de Compostela. 73 Studia Zamorensia, Salamanca. 74 Ars Praehistorica, Sabadell. 75 Bajo Aragón. Prehistoria, Zaragoza. 76 Lancia, León. $77 \mathrm{Me}$ morias de la Real Academia de la Historia, Madrid. 78 Boletín de la Real Sociedad Española de Historia Natural, Madrid. 79 Revista de Investigación, Soria. 80 Serie Universitaria de la Fundación Juan March, Madrid. 81 Monografías del Museo Arqueológico de Valladolid. $82 \mathrm{Sa}$ lamanca. Revista Provincial de Estudios.

en las citas a revistas. Aunque puede existir un solapamiento entre estos dos factores, comparando las columnas $\mathrm{C}$ y $\mathrm{D}$ del cuadro 12 podemos tratar de aislar su importancia relativa. Las diferencias apreciables en la distribución de las revistas según este criterio respecto a la del cuadro 7 se deben al número de documentos manejados.

La columna $\mathrm{C}$ indica el grado de generalización de una publicación - las cifras más altas corresponden a las revistas más especializadas- y la columna D su orientación: valores altos indican que la especialización es territorial, y bajos, temática.

Los resultados son coherentes con los que se han venido definiendo hasta ahora.

\begin{tabular}{|l|r|r|r|r|}
\hline & $\mathrm{A}$ & $\mathrm{B}$ & $\mathrm{C}$ & $\mathrm{D}$ \\
\hline Cypsela & 798 & 218 & 27,3 & 100 \\
\hline B. M. de Z. & 138 & 28 & 20,3 & 100 \\
\hline Empúries & 1.327 & 133 & 10 & 100 \\
\hline Q.P.C. & 609 & 22 & 3,6 & 100 \\
\hline EAA & 788 & 84 & 10,7 & 77,4 \\
\hline Pyrenae & 732 & 56 & 7,6 & 77 \\
\hline APL & 1.325 & 162 & 12,2 & 76 \\
\hline Munibe & 1.173 & 270 & 23 & 68 \\
\hline Caesaraugusta & 476 & 30 & 6,3 & 60 \\
\hline TP & 3.268 & 298 & 9,1 & 46,6 \\
\hline BSAA & 919 & 39 & 4,2 & 46 \\
\hline AEspA & 1.319 & 39 & 3 & 36 \\
\hline CuPAUAM & 1.058 & 48 & 4,5 & 23 \\
\hline Zephyrus & 3.108 & 506 & 16,3 & 22,9 \\
\hline EAE & 1.014 & 15 & 1,5 & 0 \\
\hline SAGVNTVM & 1.013 & 11 & 1,08 & 0 \\
\hline
\end{tabular}

Cuadro 12. A: Total de citas extraído del cuadro 1. B: Total de los cuadros 9, 10 y 11. C: Porcentaje de B sobre A. D: Porcentaje de citas a revistas de la propia Comunidad sobre $\mathrm{B}$.

Todas las revistas citadas por Cypsela y Empúries (Cuadro 9) están publicadas en Cataluña pero, mientras en la primera representan más de la cuarta parte, en la segunda sólo son el $10 \%$.

En Zephyrus el valor relativamente elevado de la columna $\mathrm{C}$ se debe, sobre todo, a la cita a revistas con una especialización temática más que espacial; por ejemplo, entre las 14 que únicamente se citan desde ella están Ars Praehistorica, el Boletín de la Real Sociedad Española de Historia Natural y la Serie Universitaria de la Fundación Juan March. Cuando sucede lo contrario se trata de publicaciones editadas fuera de Castilla-León como Gallaecia, Mainake o Bajo Aragón. Prehistoria. En consecuencia el porcentaje de citas a revistas de la propia Comunidad es de los más bajos.

La proporción significativa de citas a revistas madrileñas por parte de Archivo Español de Arqueología, Cuadernos de Prehistoria y Arqueología de la Universidad Autónoma de Madrid y Trabajos de Prehistoria corresponde a medios de difusión de instituciones de ámbito nacional. 


\section{FACTOR DE IMPACTO}

Uno de los parámetros reconocidos internacionalmente en los estudios bibliométricos de

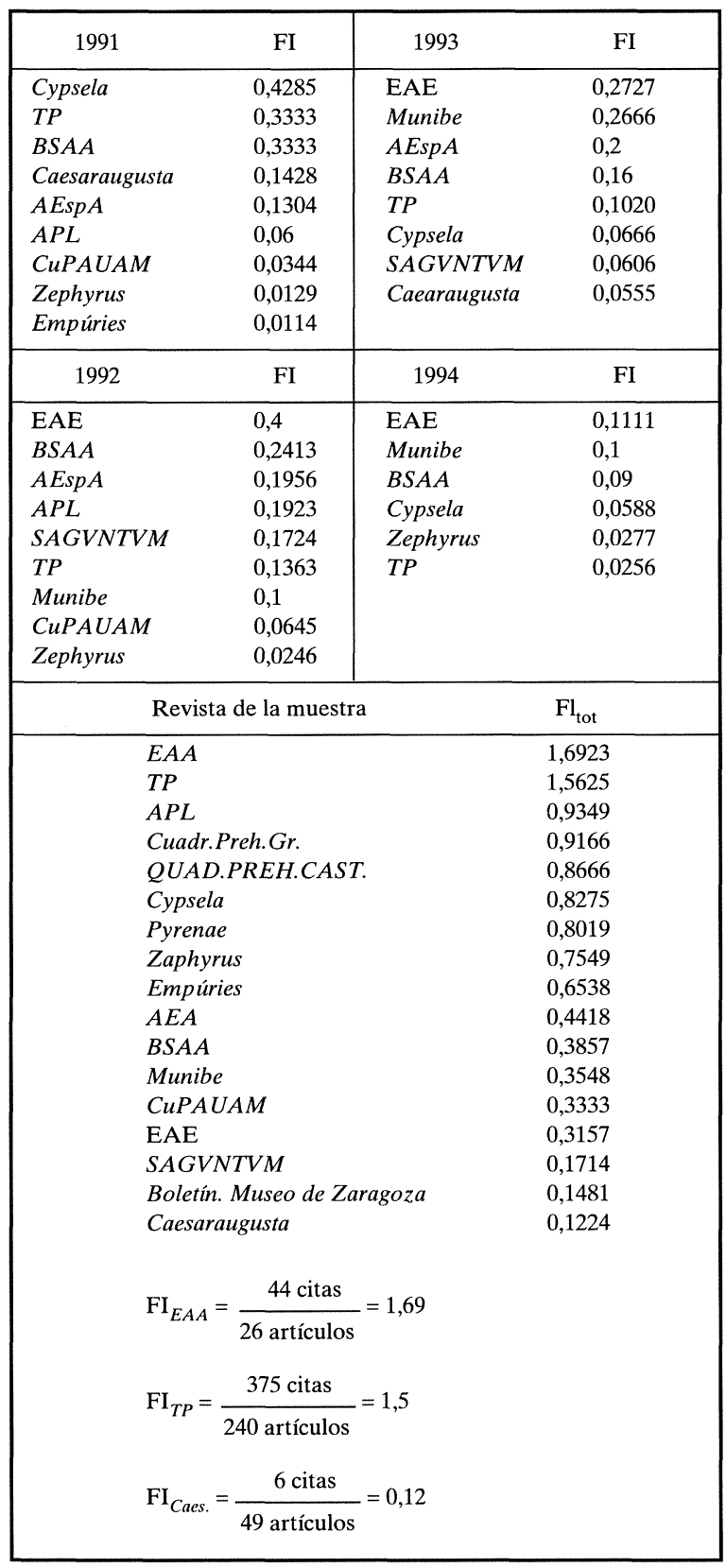

Cuadro 13. Factores de impacto (FI) de los años 1991, 1992, 1993, 1994 y global con ejemplos del cálculo del FI de Estudios de Arqueología Alavesa, Trabajos de Prehistoria y Caesaraugusta. calidad científica de publicaciones periódicas es el Factor de Impacto (FI). Mide la frecuencia con la que un artículo cualquiera de una revista ha sido citado en un año particular. Básicamente, es la proporción entre citas y artículos citables recientemente publicados. Esto es, el FI de la revista $\mathrm{X}$ se calcularía dividiendo el número de citas recibidas en un año por los artículos publicados durante los dos años anteriores en la revista $X$, por el número de artículos publicados en la revista $X$ en esos dos años.

El FI es útil para matizar el significado del número de citas absolutas. Tiende a disminuir la ventaja que en los "rankings" tienen las revistas grandes sobre las pequeñas, o las revistas de periodicidad larga sobre las que la tienen corta, y de las revistas más antiguas sobre las más nuevas. A igualdad de condiciones cuanto mayor sea el cuerpo citable, más a menudo se citará una revista. Sin embargo, al no haberse obtenido, que sepamos, el FI para revistas de Humanidades, y en concreto para Prehistoria y Arqueología, desconocíamos a priori el grado de fiabilidad de dicho parámetro.

Sabemos que cualquier análisis de citas basado únicamente en un número y tipo limitado de fuentes debe mirarse con recelo (Line, 1979), y que ni siquiera, para algunas ciencias (Física, por ejemplo), existe un grado de correlación satisfactorio entre el FI y la opinión de los expertos (Gordon, 1982: 56).

Es obvio que el FI varía según la muestra de revistas vaciadas que aportan las citas. Por eso, en nuestro caso, al ser únicamente 16 revistas y una serie monográfica las analizadas, sólo podrá hablarse de una aproximación al mismo. Además, la absoluta disparidad manifiesta en la edición de las revistas españolas de esta materia dificulta, cuando no invalida, la obtención del FI en muestras pequeñas. En efecto, hay números de revista que publican un solo artículo en un año y otras 87; revistas que no se editan durante tres años consecutivos y acumulan en un volumen posterior toda su producción, etc. hacen prácticamente imposible comparar con un mínimo de credibilidad sus FI. Es decir, una revista no editada durante un año disminuye a la mitad sus posibilidades de ser citada. Por eso, además del FI estándar obtenemos el derivado de considerar las citas totales a los ar-

T. P., 53, n. ${ }^{\circ} 1,1996$ 
tículos publicados por las revistas en los últimos cinco años, esto es:

$$
F I_{\text {tot }}=\frac{\text { Número de citas recibidas a los artículos publicados en los años de la muestra }}{\text { Número de artículos publicados en la muestra }}
$$

Por otro lado, dado el comportamiento espacial observado y analizado en el epígrafe anterior, se presenta otro tipo de lecturas que debemos mencionar. El mayor volumen de las citas a las revistas de una Comunidad Autónoma se produce desde revistas de la misma Comunidad cuando no desde ella misma (autocitas). Por eso, cuando se acumula la edición de dos años seguidos de varias revistas de una Comunidad, aumenta su FI proporcionalmente con el resto de revistas. El caso no es nuevo. Nederhof y Zwaan (1991: 332) recogen literalmente: "A causa de su fuerte orientación local, algunas disciplinas de las Ciencias Sociales y Humanidades pueden tener' sólo impacto local, y carecer completamente de impacto internacional."

En nuestro caso, como ocurre también en Sociología (Gordon, 1982: 56), se observa un paralelismo significativo entre la evaluación subjetiva de expertos, y el número total de citas a revistas, mientras que dicho paralelismo se desdibuja al comparar la evaluación subjetiva con el FI. Nuestros datos por tanto, apuntan a considerar como indicador válido para la evaluación cualitativa de las revistas españolas de Arqueología y Prehistoria, el que considera globalmente el monto total de las citas recibidas por cada revista en un período de tiempo significativo. Pero creemos que deberían seguir obteniéndose los factores de impacto durante más años y con más revistas, para poder decidir más definitivamente sobre la validez de dicho factor en la evaluación cualitativa de las revistas de esta área.

Todas estas consideraciones deben reseñarse antes de aceptar como significativos los datos que recogemos a continuación (Cuadro 13). Es decir, deben relativizarse los FI que aportamos, hasta compararlos con los obtenidos en muestras más numerosas de revistas y años vaciados.

\section{CONCLUSIONES}

Los trabajos bibliométricos aplicados a las Humanidades en España deben potenciarse no sólo para distintos procesos de evaluación sino para profundizar en el conocimiento científico de las materias a las que se refieren. En el caso de la Arqueología y Prehistoria españolas la falta de normalización que observan autores y editores dificulta los estudios de citas. Este hecho, junto al incumplimiento casi sistemático de la periodicidad de las revistas, influye también en su difusión nacional e internacional. Confiamos que este artículo sirva de llamada de atención sobre la urgente necesidad de superar este grave problema.

En la investigación cuyos resultados hemos presentado existe una correlación manifiesta entre la opinión de los expertos y el "ranking" de revistas obtenido por la cuantificación de las citas que reciben salvo, quizá, en alguna revista de muy reciente aparición. Ello justifica la utilización de este último parámetro en estudios de calidad y pone de manifiesto lö înfundado de las reticencias que algunos investigadores del ámbito de las Humanidades y las Ciencias Sociales todavía mantienen respecto a la bibliometría como forma de evaluación de la producción científica. Sin embargo, la validez del FI, obtenido con las limitaciones apuntadas, debe relativizarse a la espera de trabajos con mayor número de revistas vaciadas.

La vida media de las publicaciones en Prehistoria y Arqueología es mayor que la de las de Ciencia y Tecnología. Ello no obsta para que las más recientes sean las más valoradas. En todos los tipos de documentos no sólo las citas se concentran en las dos últimas décadas, sino que suponen aquí más de la mitad del total. Además la valoración de la vida media del documento ha de tener en cuenta los desfases entre la fecha de envío de un original para su publicación, la de su aparición real - como se ha dicho, no siempre coincidente con la que consta en el volumen- y la de su distribución (Rovira, 1994: 65). Estos desfases tienen a su vez efectos sobre la propiedad intelectual.

Los arqueólogos españoles manejan, preferentemente, publicaciones editadas en España, pero las citas extranjeras rondan el $40 \%$ del total de referencias. Los 837 títulos diferentes citados cuantifican la dispersión de las publicaciones en Prehistoria y Arqueología. Para valorar su significación recordemos que 324 sólo se 
citaron una vez y las 34 que tienen más de 100 citas (Anexo) acumulan el 63\% del total. Por otro lado, muchas han dejado de publicarse. Ahora bien, aunque este núcleo de publicaciones baste para caracterizar la producción científica en estas disciplinas, una historia de la ciencia que se restringiera a ese núcleo podría dejar de lado parte de lo que esa producción debe a la especialización. En nuestro estudio hemos advertido la importancia del territorio, bien sea el de la propia Comunidad Autónoma u otro y de la temática, fundamentalmente cronológica.

La importancia del estudio del pasado por períodos ya ha sido apuntada en otras ocasiones (Rodríguez et alii, 1993: 14) y se advierte en la baja presencia de artículos de síntesis (Fig. 7B). En cuanto al territorio, el sesgo localista revelado por las citas y la preferencia por los artículos de carácter tipológico refuerzan nuestras conclusiones sobre la distribución territorial de las revistas, revelan la orientación descriptiva de la investigación y sugieren aspectos de la sociología de la ciencia.

La multiplicación de revistas durante los últimos cincuenta años se conecta con "la profesionalización, cada vez mayor, que adquiere la arqueología" (Salvatierra, 1990: 25) sobre la que se superpone la voluntad de las instituciones editoras de facilitar un vehículo de difusión a la investigación propia. Esto implica, simultáneamente, unas ciertas dificultades de publicación para los autores que no pertenecen a esas instituciones. Una alternativa ha sido la creación de revistas de ámbito restringido o la publicación en revistas de estudios locales volviendo a una estrategia que definió parte de la producción científica durante el primer tercio de siglo (Salvatierra, 1990: 21-22). Otro factor de crecimiento es el papel que juegan en el acrecentamiento de bibliotecas por intercambios.

Algunos de los aspectos negativos de este panorama editorial se refieren a la dificultad de contar tanto con un conocimiento exhaustivo de todas las publicaciones como con la financiación e infraestructura necesarias para su adquisición y gestión. Estos aspectos ponen de relieve la extraordinaria importancia de las bases de datos para la investigación.
Pero el rasgo más preocupante que hemos observado es de carácter metodológico. El localismo expresa una orientación descriptiva en la investigación arqueológica que reduce las posibilidades de generalización, de elaboración de síntesis y de reflexión teórica. De esta manera, cabe entender la conexión entre la cita a una Comunidad y la existencia de publicaciones editadas en ella. En ausencia de éstas, la representación de ese territorio en la bibliografía suele restringirse a citas aisladas en las de carácter general más o menos numerosas según la tradición de investigación en el mismo (compárense Galicia y Murcia). Además, las interpretaciones del pasado tienen como referencia unidades administrativas actuales como municipios, provincias, comarcas, siendo este un rasgo común a todas las publicaciones con independencia de su lugar de edición.

El problema no es que se delimite de esta manera la zona de estudio, sino que este sesgo actualista condicione la propia conceptualización del problema histórico en Prehistoria y Arqueología. Las unidades administrativas actuales se proyectan al pasado como si hubieran existido desde siempre, y se estudian manejando una documentación preferentemente local. Esta falla metodológica conlleva el riesgo de caer en un provincianismo y autonomismo a ultranza por desconocimiento y aislamiento que puede facilitar interesadas tergiversaciones del pasado.

$\mathrm{El}$ análisis bibliométrico facilita datos explícitos y comparables para evaluar estas cuestiones.

\section{AGRADECIMIENTOS}

La realización de este artículo se ha visto enormemente facilitada por la colaboración que nos prestó en todo momento el personal de la biblioteca del Museo Arqueológico Nacional de Madrid ("Colección de Prehistoria" del CSIC y Ministerio de Cultura).

\section{BIBLIOGRAFÍA}

Авт, H.A. (1992): "Publication practices in various sciences". Scientometrics, 24: 441-447.

FERreiro AlÁEZ, L. (1993): "Bibliometría (análisis bivariante)". Ed. EYPASA. Madrid.

T. P., 53, n. ${ }^{\circ} 1,1996$ 
FinKenstaedT, T. (1990): "Measuring research performance in the humanities". Scientometrics, 19: 409-417.

GoRDON, M.D. (1982): "Citation ranking versus subjective evaluation in the determination of journal hierarchies in the Social Sciences". Journal of the American Society for Information Science, January, 55-57.

GRAN-AYMERICH, E. Y GRAN-AYMERICH, J. (1991): "Les échanges franco-espagnols et la mise en place des institutions archéologiques (1830-1839)". En J. Arce y R. Olmos (coords.): "Historiografía de la Arqueología y de la Historia Antigua en España (siglos XVIII-XX)". Ministerio de Cultura. Madrid: 117-124.

GrünHAGEN, W. (1979): "Zur Geschichte der Abteilung Madrid des Deutschen Archäologischen Instituts von 1929 bis 1979". Das Deutsche Archäologische. Institut Geschichte und Dokumente, 3: 117-170.

LiNE, M.B. (1979): "The influence of the type of sources used on the results of citation analyses". Journal of Documentation, 35(4): 265-284.

Nederhof, A.J. y ZWAAN, R.A. (1991): "Quality judgments of journals as indicators of research performance in the Humanities and the Social and Behavioral Sciences". Journal of the American Society for Information Science, 42(5): 332-340.

Remesal, José; GonzÁlez, Eulalia; Martínez, Francisco Javier; MuÑoz, M. ${ }^{a}$ Jesús; Orejas, ALmudena; Ribagorda, Miguel y Utasá, Cristina (1991): "La presencia de la Historiografía sobre Historia Antigua Española en algunas revistas extranjeras". En J. Arce y R. Olmos (coords.): Historiografía de la Arqueología y de la Historia Antigua en España (siglos $X V I I I-X X$ ). Ministerio de Cultura. Madrid: 219-225.

Rodríguez Alcalde, Ángel L.; San Millán Bujanda, M. ${ }^{a}$ Jesús; SÁnchez Nistal, José María; Chapa Brunet, Teresa; Martínez NAVARrete, M. ${ }^{a}$ IsABel y RuiZ-ZaPATERO, GonZalo (1993): "Análisis bibliométrico de Trabajos de Prehistoria: un chequeo a la Prehistoria española de las tres últimas décadas". Trabajos de Prehistoria, 50: 11-37.
Rodríguez Alcalde, Ángel L.; SÁnChez Nistal, José María; Martínez Navarrete, $M^{a}$. Is Abel; SAN Millán Bujanda, M. ${ }^{a}$ Jesús (e.p.): "El análisis bibliométrico como aportación a la historiografía. Las citas en Prehistoria y Arqueología". II Congreso Internacional de Historiografía de la Arqueología en España (siglos XVIII-XIX): La cristalización del pasado, génesis y desarrollo del marco institucional de la Arqueología en España (Madrid, noviembre, 1995).

Rovira LlORÉNS, SALVADOR (1994): "Estudio bibliométrico del Boletín de la Asociación Española de Amigos de la Arqueología". Boletín de la Asociación Española de Amigos de la Arqueología, 34, Enero-Diciembre: 57-64.

RUIZ-ZAPATERO, GONZALO (1993): "La organización de la Arqueología en España". En M. ${ }^{a}$ Isabel Martínez Navarrete (coord.): Teoría y práctica de la Prehistoria: perspectivas desde los extremos de Europa. Universidad de Cantabria, CSIC. Santander: 45-73.

Sales Heredia, P.; García Marín, A. y Román ROMÁN, A. (e.p.): "Las revistas científicas, transmisoras del conocimiento. Una metodología de evaluación". Ponencia en ECONSID'95. II Encuentro de organización del conocimiento en sistemas documentales $y$ de información (Madrid, noviembre, 1995).

Salvatierra Cuenca, Vicente (1990): “Cien años de Arqueología Medieval. Perspectivas desde la periferia: Jaén". Universidad de Granada. Granada.

SÁNCHEZ NiSTAL, J.M. (1995): "La geografía española a través de sus revistas. Una aproximación bibliométrica". Estudios Geográficos, LVI, 220: 547-621.

SCHubART, H. (1995): "Discurso de bienvenida del director del Instituto Arqueológico Alemán de Madrid Prof. Dr. Dr. h. c. Hermanfrid Schubart". Madrider Mitteilungen, 36: XIX-XXIV.

Singleton, A. (1976): "Journal ranking and selection: a review in Physics". Journal of Documentation, 32(4): 258-289.

Villagrá Rubio, A. (1992): "Scientific Production of Spanish universities in the fields of Social Sciences and Language”. Scientometrics, 24: 3-19. 
Anexo. "RANKING” TOTAL DE REVISTAS Y SERIES MONOGRÁFICAS. Publicaciones con 30 o más citas recibidas: número de citas (número de autocitas de revista) [también como]

961 (339) Trabajos de Prehistoria. Madrid.

934 (143) Archivo Español de Arqueología. Madrid.

882 (82) Excavaciones Arqueológicas en España. Madrid.

846 Noticiario Arqueológico Hispánico. Madrid.

724 (145) Empúries. Barcelona.

679 (243) Zephyrus. Salamanca.

537 (110) Boletín del Seminario de Estudios de Arte y Arqueología. Valladolid

523 (305) Munibe. San Sebastián.

485 (147) Archivo de Prehistoria Levantina. Valencia.

432 (151) Cuadernos de Prehistoria de la Universidad de Granada. Granada.

432 (87) Saguntum. Papeles de Laboratorio de Arqueología de Valencia. Valencia.

431 Madrider Mitteilungen. Mainz.

404 Serie de Trabajos. Servicio de Investigación Prehistórica. Valencia.

394 (154) Estudios de Arqueología Alavesa. Vitoria.

386 Bibliotheca Praehistorica Hispana. Madrid.

360 (43) Pyrenae. Barcelona.

356 Informes y Memorias de la Comisaría General de Excavaciones Arqueológicas. Madrid.

258 Revista de Arqueología. Madrid.

237 (98) Cuadernos de Prehistoria y Arqueología Castellonenses. Castellón.

200 (90) Cypsela. Girona.

185 Habis. Sevilla.

172 Boletín de la Real Academia de la Historia. Madrid.

162 Príncipe de Viana. Pamplona.

161 Trabajos de Arqueología Navarra. Pamplona. [Arqueología Navarra].

160 (15) Casaraugusta. Zaragoza.

159 Studia Archaeologia. Valladolid.

143 (31) Cuadernos de Prehistoria y Arqueología de la Universidad Autónoma de Madrid. Madrid.

129 Revista de Archivos, Bibliotecas y Museos. Madrid.

126 Anuari de l'Institut d'Estudis Catalans. Barcelona

126 Lucentum. Alicante.

122 Huelva Arqueológica. Huelva.

121 Boletín de la Asociación Española de Amigos de la Arqueología. Madrid.

111 Anuario Arqueológico de Andalucía. Sevilla.

103 Memorias de los Museos Arqueológicos Provinciales. Madrid.

93 Actas y Memorias de la Sociedad Española de Antropología, Etnografía y Prehistoria. Madrid.

93 Informaciò Arqueologica. Barcelona.

92 Acta Arqueológica Hispánica. Madrid.

92 Mélanges de la Casa de Velázquez. Madrid.

86 Memorias de la Comisión de Investigaciones Paleontológicas y Prehistóricas. Madrid.

85 Fonaments. Barcelona.

82 Wad-Al-Hayara. Guadalajara.

81 Annals de l'Institut d'Estudis Gironins. Girona.

78 Monografías Arqueológicas Universidad de Zaragoza. Zaragoza.

74 Kobie. Bilbao.

74 Publicaciones Eventuales del Instituto de Arqueología y Prehistoria. Barcelona.

73 Butlletí Arqueologic. Tarragona.

73 Emerita. Madrid.

72 Tribuna d'Arqueologia. Barcelona.
71 Arqueología Espacial. Teruel.

71 Revista de Girona. Girona.

67 Cuadernos de Estudios Gallegos. Santiago de Compostela.

67 Monografías del Centro de Investigación y Museo de Altamira. Santander.

66 Veleia. Vitoria.

64 Excavaciones en Navarra. Pamplona.

63 Boletín. Museo de Zaragoza. Zaragoza.

63 Numantia. Soria.

62 Investigación Arqueológica-Arkeoikuska. Vitoria.

60 Serie Arqueológica. Universidad de Valencia. Valencia.

58 Excavacions Arqueologiques a Catalunya. Barcelona.

57 Oretania. Linares.

56 Boletín del Instituto de Estudios Asturianos. Oviedo.

54 Boletín de la Real Sociedad Española de Historia Natural. Madrid.

54 Saitabi. Valencia.

53 Monografies Emporitanes. Barcelona.

52 Boletín de la Sociedad Castellonense de Cultura. Castellón.

51 Celtiberia. Soria.

50 Anales de Prehistoria y Arqueología. Murcia.

50 Boletín de la Institución Sancho el Sabio. Vitoria.

49 Bolletí de la Societat Arqueologica Luliana. Palma de Mallorca.

49 Memorias del Seminario de Prehistoria y Arqueología. Salamanca.

49 Serie Monográfica. Centre d'Investigacions Arqueologiques. Girona.

48 Numisma. Madrid.

48 Teruel. Teruel.

47 Bajo Aragón. Prehistoria. Zaragoza.

47 Boletín del Instituto de Estudios Giennenses. Jaén.

47 Revista de Estudios Extremeños. Badajoz.

46 Acta Salmanticensia. Salamanca.

46 Arqueología. Ministerio de Cultura. Madrid.

46 Boletín del Museo Arqueológico Nacional. Madrid.

46 Cuadernos de Arqueología de Deusto. Bilbao.

46 Trabajos del Museo Arqueológico de Ibiza.

45 Sautuola. Santander.

43 Estudios de Prehistoria y Arqueología Madrileñas. Madrid.

43 Gerion. Madrid.

42 Mainake. Málaga.

40 Ars Praehistorica. Sabadell.

40 Ilerda. Lleida.

39 Cota Zero. Barcelona.

37 Cuadernos de Sección. Antropología/Etnografía, Prehistoria/Arqueología. San Sebastián.

37 Speleon. Oviedo.

36 Cuadernos de Investigación Prehistórica. Brocar. Logroño.

36 Revista de la Universidad Complutense. Madrid.

35 Baetica. Málaga.

35 Boletín Auriense. Ourense.

35 Hispania Antiqua. Revista de Historia Antigua. Valladolid.

34 Acta Numismatica. Barcelona.

34 Cuadernos de Historia Primitiva. Madrid.

34 Italica. Sevilla.

34 Memorias de la Real Academia de la Historia. Madrid.

34 Programa de Investigaciones Protohistóricas. Barcelona.

33 Aula Orientalis. Sabadell.

33 Kalathos. Teruel.

32 Gallaecia. Santiago de Compostela.

32 Publicaciones de la Institución Tello Téllez de Meneses. Palencia.

32 Revista del Instituto de Estudios Alicantinos. Alicante.

31 Memòries d'Escavaciò. Taller Escola d'Arqueologia. Tarragona.

30 Bolskan. Huesca.

T. P., 53, n. ${ }^{\circ} 1,1996$ 